\title{
A neighborhood for complex job shop scheduling problems with regular objectives
}

\author{
Reinhard Bürgy
}

Accepted for publication in Journal of Scheduling (April 2017)

The final publication is available at Springer

via http://dx.doi.org/10.1007/s10951-017-0532-2

\begin{abstract}
Due to the limited applicability in practice of the classical job shop scheduling problem, many researchers have addressed more complex versions of this problem by including additional process features, such as time lags, setup times, and buffer limitations, and have pursued objectives that are more practically relevant than the makespan, such as total flow time and total weighted tardiness. However, most proposed solution approaches are tailored to the specific scheduling problem studied and are not applicable to more general settings.

This article proposes a neighborhood that can be applied for a large class of job shop scheduling problems with regular objectives. Feasible neighbor solutions are generated by extracting a job from a given solution and reinserting it into a neighbor position. This neighbor generation in a sense extends the simple swapping of critical arcs, a mechanism that is widely used in the classical job shop but that is not applicable in more complex job shop problems.

The neighborhood is embedded in a tabu search, and its performance is evaluated with an extensive experimental study using three standard job shop scheduling problems: the (classical) job shop, the job shop with sequence-dependent setup times, and the blocking job shop, combined with the following five regular objectives: makespan, total flow time, total squared flow time, total tardiness, and total weighted tardiness. The obtained results support the validity of the approach.
\end{abstract}

Keywords job shop scheduling $\cdot$ general regular objective $\cdot$ sequence-dependent setup times $\cdot$ blocking job shop $\cdot$ job insertion $\cdot$ neighborhood $\cdot$ tabu search

\section{Introduction}

The classical job shop scheduling problem has attracted the attention of a large number of researchers and has earned the reputation of being one of the most computa- 
tionally stubborn combinatorial optimization problems (Applegate and Cook, 1991). This standard scheduling problem is, however, of limited applicability in practice since real-world scheduling problems possess process features that are not captured by the classical job shop model, such as, for example, sequence-dependent setup times, minimum and maximum time lags between operations, limited buffer capacity, routing flexibility, and transportation tasks. Also, the makespan is not the only relevant performance measure in practice: total (weighted) flow time, total squared tardiness, and other possibly nonlinear measures of earliness and tardiness are equally relevant in applications. This has given rise to active research on job shop scheduling models that better capture real-world scheduling problems (Potts and Strusevich, 2009). Some of these models have established themselves over the years as standard problems in the literature, among them the job shop with total weighted tardiness objective, the job shop with sequence-dependent setup times, the no-wait job shop, and the blocking job shop.

Referring to the general area of scheduling research, Potts and Strusevich (2009) mention that many researchers were motivated by the need to create scheduling models capturing more features that arise in practice. However, they conclude that "In spite of the vast body of research being produced, a large gap still remains between theory and practice." A similar assessment can be found in (Pinedo, 2012, pp. 431 435). This gap has also been widely acknowledged in scheduling problems of the job shop type.

A main reason for the existence of this gap is, in our view, the lack of research into generic job shop scheduling problems that would model a wide range of process features and cover generic objectives. Indeed, most articles study specific job shop problems and include only a few additional features. As a result, the proposed solution approaches are highly specialized, and job shop scheduling research has become fragmented. Bülbül and Kaminsky (2013) concur with this opinion, and point out that, as a result, job shop scheduling software is highly specialized and customized.

The following are some of the few contributions addressing more general job shop scheduling problems. Mati et al (2011) considered the job shop problem with a general regular objective. This class of objectives comprises all functions that are monotone non-decreasing in the completion times. They formulated the problem in a disjunctive graph and developed a local search heuristic where neighbors are built by swapping a critical arc. For future research, they proposed investigating more complex job shop scheduling problems with a general regular objective. Bülbül and Kaminsky (2013) developed a decomposition heuristic for the job shop problem applicable to all linear functions of the operation start (and end) times. This class of objectives makes it possible to consider, for example, intermediate holding costs, which are a relevant performance measure in practice. They formulated the problem in a disjunctive graph and developed a shifting-bottleneck-based solution approach. An interesting and novel component of their method is the integration of dual values from the timing problems (of partial schedules) into the single machine subproblems. Grimes and Hebrard (2015) recently considered a generic disjunctive machine scheduling problem in which various processing features and objectives can be modeled. They proposed a constraint programming approach that combines a number of generic search techniques (restarts, adaptive heuristics, and solution-guided 
branching). While the basic approach is generic, some specific knowledge is incorporated to improve its effectiveness in certain problem types. Their numerical tests on some standard job shop problems showed that the approach works well for small and medium-sized instances, but is less suited for solving large instances. In addition, they observe that a good initial upper bound improves the performance of the approach.

In view of the excellent (hybrid) local search approaches developed for specific job shop scheduling problems (see, e.g., Nowicki and Smutnicki, 2005; Zhang et al, 2008; González et al, 2012a), it seems surprising that almost no local search methods have been proposed for a more general job shop scheduling setting. In fact, the applied meta-heuristics are typically generic in nature; however, the neighborhood, a main component of the local search, is in most cases only applicable in a rather narrow setting. The generation of feasible neighbors is a major problem. Indeed, the standard strategy of swapping critical arcs generates a feasible neighbor with certainty in the (classical) job shop, but may lead to an infeasible neighbor in other variants. For the job shop with sequence-dependent setup times, necessary conditions for feasibility have been established (see, e.g., Vela et al, 2010), and a critical arc is only swapped if such a condition is fulfilled. However, in more complex job shop scheduling problems, such as the blocking job shop, swapping a single arc mostly leads to an infeasible solution. Hence, another approach is needed for these complex scheduling problems.

In this paper, we develop a neighborhood that is applicable to a large class of job shop problems and any regular objective. The neighborhood in a sense extends the idea of swapping a critical arc and is based on a neighbor generation scheme that extracts a job from a given solution and reinserts it into a feasible neighbor position. The neighbor generation scheme relies on the job insertion theory developed by Gröflin and Klinkert (2007), and has already been successfully applied in various job shop scheduling problems with the makespan objective, namely, in the (flexible) blocking job shop (Gröflin and Klinkert, 2009; Gröflin et al, 2011), in the blocking job shop with rail-bound transportation (Bürgy and Gröflin, 2016), and in a more general job shop model (Bürgy, 2014). This article casts the neighbor generation scheme in a unifying framework and extends its use to any regular objective. Note that we abstained from dealing with machine flexibility in this work and refer to (Gröflin et al, 2011; Bürgy, 2014) for a possible inclusion of this feature.

The remainder of the article is organized as follows. The next section proposes a generic job shop scheduling problem called the complex job shop with a regular objective (CJS-R). Sect. 3 introduces the job insertion problem, which is an important subproblem of the CJS-R problem. The feasible solutions of this subproblem are characterized as the stable sets (with a prescribed cardinality) of a conflict graph, and structural properties of this conflict graph are established. Based on these results, Sect. 4 provides a generic scheme for deriving feasible neighbor solutions and proposes a specific neighborhood. Sect. 5 casts this neighborhood in a tabu search, whose performance is evaluated with an extensive experimental study on some wellknown, standard job shop scheduling problems. Proofs and detailed numerical results are provided in the Appendix. 
The introduction is concluded with some notation and terminology. Unless otherwise stated, the graphs will be directed and simple, and the following standard notation will be used. In a graph $G=(V, E)$, an arc $e=(v, w) \in E$ has a tail $t(e)=v$ and a head $h(e)=w$. For any set $V^{\prime} \subseteq V$, the set of its ingoging $\operatorname{arcs}$ is $\delta^{-}\left(V^{\prime}\right)=\{e \in E$ : $t(e) \in V \backslash V^{\prime}$ and $\left.h(e) \in V^{\prime}\right\}$, the set of its outgoing arcs is $\delta^{+}\left(V^{\prime}\right)=\left\{e \in E: t(e) \in V^{\prime}\right.$ and $\left.h(e) \in V \backslash V^{\prime}\right\}$, the set of arcs in its cut is $\delta\left(V^{\prime}\right)=\delta^{-}\left(V^{\prime}\right) \cup \delta^{+}\left(V^{\prime}\right)$, and the set of arcs with both ends in $V^{\prime}$ is $\gamma\left(V^{\prime}\right)=\left\{e \in E: t(e) \in V^{\prime}\right.$ and $\left.h(e) \in V^{\prime}\right\}$. If an arc length vector $c \in \mathbb{R}^{E}$ is given, $G$ will be denoted by the triplet $G=(V, E, c)$. In $G=(V, E, c)$, a cycle is called positive if its length is positive.

\section{The complex job shop scheduling problem with a regular objective}

We first propose a generic job shop scheduling problem called the complex job shop with a regular objective (CJS-R). The CJS-R problem is based on the scheduling model presented in (Gröflin and Klinkert, 2007), which is adapted to incorporate jobs and regular objectives. The CJS-R problem is then formulated as a combinatorial problem in a disjunctive graph. Finally, two standard job shop scheduling problems, the job shop problem with sequence dependent setup times and a regular objective and the blocking job shop with transfer times, setup times, and a regular objective, are specified as CJS-R problems.

\subsection{Notation, data, and a problem formulation}

Let $V$ be a finite set of events (e.g., start or end of operations), $\sigma \in V$ a dummy start event, and $V^{-}=V \backslash\{\sigma\}$. Let $\mathscr{J}$ be a set of jobs such that $\mathscr{J}$ forms a partition of $V^{-}$, i.e., a job $J \in \mathscr{J}$ consists of a set of events and each event $v \in V^{-}$belongs to exactly one job. For each job $J \in \mathscr{J}$, the set $V_{J} \subseteq V$ contains all events that belong to $J$. For ease of notation, introduce function $J o b: V \backslash\{\sigma\} \rightarrow \mathscr{J}$ mapping any event to its job, i.e., if event $v$ belongs to job $J$ then $J o b(v)=J$.

Furthermore, let $A \subseteq V \times V^{-}$and $E \subseteq V^{-} \times V^{-}$be two disjoint sets of precedence constraints and $d \in \mathbb{R}^{A \cup E}$ a weight function. A precedence constraint $(v, w) \in A \cup E$ with weight $d_{v w}$ states that event $v$ must occur at least $d_{v w}$ time units before event $w$. Elements of set $A$ and $E$ are called conjunctive and disjunctive precedence constraints, respectively. Each conjunctive precedence constraint must be satisfied in any feasible solution while a disjunctive precedence constraint must hold if some other disjunctive constraint in $E$ is violated. To formalize this disjunctive structure, define a family of disjunctive sets $\mathscr{E} \subseteq 2^{E}$ where $\mathscr{E}$ is a partition of $E$ into pairs. Then, for each (unordered) pair $\left\{(v, w),\left(v^{\prime}, w^{\prime}\right)\right\} \in \mathscr{E}$, either precedence constraint $(v, w)$ or $\left(v^{\prime}, w^{\prime}\right)$ must be fulfilled in any feasible solution. Note that a general disjunctive pair is sometimes denoted by $\{e, \bar{e}\}$ and $\bar{e}$ is called the mate of $e$.

Let $\alpha=\left(\alpha_{v} \in \mathbb{R}: v \in V\right)$ be a vector of the times $\alpha_{v}$ at which events $v \in V$ occur. Vector $\alpha$ specifies a schedule. The objective function $f: \mathbb{R}^{V} \rightarrow \mathbb{R}$ considered here satisfies:

$$
\alpha \leq \alpha^{\prime} \text { implies } f(\alpha) \leq f\left(\alpha^{\prime}\right) \text { for all vectors } \alpha, \alpha^{\prime} \in \mathbb{R}^{V} \text {. }
$$


Objective functions satisfying (1) are called regular (see, e.g., Pinedo, 2012, p. 19). The CJS-R problem $(V, \mathscr{J}, A, E, \mathscr{E}, d, f)$ can then be formulated as the following disjunctive programming problem:

$$
\text { minimize } f(\alpha), \alpha \in \mathbb{R}^{V}
$$

subject to:

$$
\begin{aligned}
& \alpha_{w}-\alpha_{v} \geq d_{v w} \text { for all }(v, w) \in A, \\
& \alpha_{w}-\alpha_{v} \geq d_{v w} \text { or } \\
& \alpha_{w^{\prime}}-\alpha_{v^{\prime}} \geq d_{v^{\prime} w^{\prime}} \text { for all }\left\{(v, w),\left(v^{\prime}, w^{\prime}\right)\right\} \in \mathscr{E}, \\
& \alpha_{\sigma}=0 .
\end{aligned}
$$

Any feasible solution $\alpha \in \mathbb{R}^{V}$ of the CJS-R specifies times $\alpha_{v}$ for all events $v \in V$ so that all conjunctive precedence constraints are satisfied (3), at least one disjunctive precedence constraint of each disjunctive pair is satisfied (4), and the start event occurs at time 0 (5). The objective (2) is to minimize the value $f(\alpha)$.

It can generally be assumed that the solution space $\left\{\alpha \in \mathbb{R}^{V}: \alpha_{\sigma}=0, \alpha_{w}-\alpha_{v} \geq\right.$ $d_{v w}$ for all $\left.(v, w) \in A\right\}$ is nonempty. According to the meaning of event $\sigma$, we also assume that $(\sigma, v) \in A$ with weight $d_{\sigma v} \geq 0$ for all $v \in V^{-}$, stipulating together with (5) that any feasible schedule $\alpha$ consists of nonnegative starting times.

In this paper, we further restrict the use of the conjunctive and disjunctive precedence constraints as follows. Each conjunctive precedence constraint considers two events of the same job or involves the dummy start, i.e., for all $(v, w) \in A$ with $v \neq \sigma$ :

$$
J o b(v)=J o b(w) .
$$

Conversely, each disjunctive precedence constraint considers two events of distinct jobs, i.e., for all $(v, w) \in E$ :

$$
J o b(v) \neq J o b(w) .
$$

Furthermore, each pair of disjunctive precedence constraints considers events that belong to exactly two distinct jobs. More precisely, for all $\left\{(v, w),\left(v^{\prime}, w^{\prime}\right)\right\} \in \mathscr{E}$ :

$$
J o b(v)=\operatorname{Job}\left(w^{\prime}\right) \text { and } J o b(w)=J o b\left(v^{\prime}\right) .
$$

Moreover, for each disjunctive pair, at most one precedence constraint can be fulfilled in any feasible solution, i.e., for all $\left\{(v, w),\left(v^{\prime}, w^{\prime}\right)\right\} \in \mathscr{E}$ :

$$
\left\{\alpha \in \mathbb{R}^{V}: \alpha_{\sigma}=0, \alpha_{q}-\alpha_{p} \geq d_{p q} \text { for all }(p, q) \in A \cup\left\{(v, w),\left(v^{\prime}, w^{\prime}\right)\right\}\right\}=\emptyset .
$$

Some remarks are in order. While there is no mention of resources in the CJS-R scheduling problem, the most common applications are scheduling problems involving machines with unit capacities. Then, the capacity constraints can easily be captured by the disjunctive constraints (4). Also, there is no prescribed job structure. In many cases, a job is just a sequence of operations, but the CJS-R problem makes it possible to specify other type of structures, such as assembly or disassembly operations. 


\subsection{A formulation in a disjunctive graph}

The CJS-R problem $(V, \mathscr{J}, A, E, \mathscr{E}, d, f)$ is formulated in an associated disjunctive graph $G=(V, A, E, \mathscr{E}, d)$ that is defined as follows. Each event $v \in V$ is represented by a node, and we identify a node with the event it represents. Each precedence constraint $(v, w) \in A \cup E$ is represented by an arc with length $d_{v w}$. Consequently, $A$ is the set of conjunctive arcs, $E$ is the set of disjunctive $\operatorname{arcs}, \mathscr{E}$ is the family of disjunctive arcs pairs, and $d$ is the arc length vector. Note that the conjunctive part $(V, A, d)$ of $G$ contains no positive cycle as there exists no feasible solution otherwise.

In order to capture solutions in graph $G$, we introduce selections of disjunctive arcs.

Definition 1 Any subset of disjunctive $\operatorname{arcs} S \subseteq E$ is called a selection. A selection $S$ is positive acyclic if its associated graph $(V, A \cup S, d)$ contains no positive cycle, and is positive cyclic otherwise. A selection $S$ is complete if $S \cap D \neq \emptyset$ for all $D \in \mathscr{E}$.

Given any selection $S \subseteq E$, we have the following timing problem at hand. The space of the feasible times for the events is

$$
\Omega(S)=\left\{\alpha \in \mathbb{R}^{V}: \alpha_{\sigma}=0, \alpha_{w}-\alpha_{v} \geq d_{v w} \text { for all }(v, w) \in A \cup S\right\} .
$$

Clearly, $\Omega(S) \neq \emptyset$ if and only if $S$ is positive acyclic. Consequently, a selection $S$ is called feasible if $S$ is complete and positive acyclic. Observe that, in a feasible selection, exactly one disjunctive arc is picked for each pair of arcs. Indeed, for all $D \in \mathscr{E}$, graph $(V, A \cup D, d)$ is positive cyclic by (9), hence $|D \cap S| \leq 1$ if $S$ is positive acyclic, therefore $|D \cap S|=1$ if $S$ is feasible.

For any feasible selection $S$, earliest times $\alpha(S)=\left(\alpha_{v}(S): v \in V\right)$ can be efficiently calculated by determining the length $\alpha_{v}(S)$ of a longest path from $\sigma$ to $v$ in graph $(V, A \cup S, d)$ for each node $v \in V$. Then, $\alpha(S) \leq \alpha^{\prime}$ for all $\alpha^{\prime} \in \Omega(S)$. As the objective function $f$ is regular,

$$
f(\alpha(S))=\min \{f(\alpha): \alpha \in \Omega(S)\},
$$

i.e., the earliest time schedule $\alpha(S)$ is an optimal solution in $\Omega(S)$.

The CJS-R problem $(V, \mathscr{J}, A, E, \mathscr{E}, d, f)$ can then be formulated as the following combinatorial problem in disjunctive graph $G$ : "Among all feasible selections, find a selection $S$ minimizing $f(\alpha(S))$."

We conclude this section by schematically illustrating the assumptions (6), (7), and (8) in Fig. 1. The conjunctive and disjunctive arcs are depicted by solid and dashed lines, respectively. Considering all conjunctive arcs not incident to $\sigma$, (6) states that both end nodes of these arcs belong to the same job. In contrast, as stated in (7), the two end nodes of a disjunctive arc must belong to different jobs. By (8), a pair of disjunctive arcs links nodes of the same two jobs, and the arcs point in opposite directions, as depicted by $e$ and $\bar{e}$. Finally, an arc $(\sigma, v)$ should be present for each $v \in V$. However, $(\sigma, v)$ may be omitted if there exists a nonnegative length path from $\sigma$ to $v$ in the conjunctive part $(V, A, d)$ as it ensures that $v$ obtains a nonnegative starting time.

In the sequel of the paper, CJS-R problems are specified by directly constructing their disjunctive graph $G=(V, A, E, \mathscr{E}, d)$. 


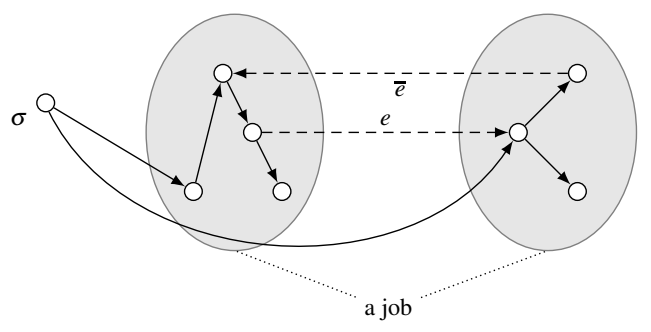

Fig. 1: Schematic illustration of the assumptions stated in (6), (7), and (8).

\subsection{Examples}

A large class of job shop problems can be formulated as CJS-R problems. We give two specific examples, which will be used in the sequel of the paper.

\subsubsection{The job shop with sequence-dependent setup times and a regular objective}

The job shop with sequence-dependent setup times and a regular objective (JSS) is a version of the classical job shop including sequence-dependent setup times and allowing all regular objectives. The problem can be formally described as follows. Given a set of operations $I$ and a set of machines $M$. Each operation $i \in I$ needs a specific machine, say $m_{i} \in M$, for its non-preemptive execution of duration $p_{i}>0$. The set of operations is structured into jobs: given is a set of jobs $\mathscr{J}^{\prime} \subseteq 2^{I}$ such that $\mathscr{J}^{\prime}$ is a partition of $I$. For each job $J \in \mathscr{J}^{\prime}$, its set of operations $\{i: i \in J\}$ is ordered $\left(J_{1}, J_{2}, \ldots, J_{|J|}\right)$ specifying in which sequence the operations of $J$ must be executed. Hereby $J_{r}$ denotes the $r$-th operation of job $J$. Two operations $i$ and $j$ of some job $J$ are said to be consecutive if $i=J_{r}$ and $j=J_{r+1}$ for some $r, 1 \leq r<|J|$.

We allow for sequence-dependent setup times. If two operations $i$ and $j$ are using the same machine $m$ and $j$ follows $i$ directly on $m$, then a setup of duration $s_{i j} \geq$ 0 occurs between the completion of $i$ and the start of $j$. We assume that the setup durations satisfy the so-called weak triangle inequality (see, e.g., Brucker and Knust, 2011 , p. 11), i.e., for any distinct operations $i, j, k$ using a same machine, $s_{i j}+p_{j}+$ $s_{j k} \geq s_{i k}$ must hold. (Otherwise setup times between non-adjacent operations on a machine might become active in the problem formulation.)

We also allow to specify a release time $s_{J}^{\mathrm{r}} \geq 0$ and a due date $s_{J}^{\mathrm{d}} \geq 0$ for each job $J \in \mathscr{J}^{\prime}$. While the release times must be satisfied in any feasible solution, violations of the due date are allowed but can be penalized in the objective function.

The problem consists in finding starting times for all operations $i \in I$ so that each machine processes at most one operation at any time and some regular objective is minimized. In the three field notation introduced by Graham et al (1979), the JSS is specified by $J\left|r_{j}, s_{i j}\right|$ reg.

The JSS can be described as a CJS-R problem in disjunctive graph $G=(V, A, E$, $\mathscr{E}, d$ ) as follows (see also the example below, which is illustrated in Fig. 2). For each operation $i \in I$, introduce a node $v_{i} \in V$ indicating the start of operation $i$. For each 
job $J^{\prime} \in \mathscr{J}^{\prime}$, introduce a job $J$ in $\mathscr{J}$ containing all nodes of the operations belonging to $J^{\prime}$.

The set of conjunctive $\operatorname{arcs} A$ is built as follows:

- For each job $J \in \mathscr{J}^{\prime}$, add an arc $\left(\sigma, v_{J_{1}}\right)$ with length $s_{J}^{\mathrm{r}}$ reflecting the release time of $J$.

- For each job $J \in \mathscr{J}^{\prime}$ and each pair of consecutive operations $i, j \in J$, add an arc $\left(v_{i}, v_{j}\right)$ with length $p_{i}$ reflecting the processing order within a job.

- For each job $J \in \mathscr{J}^{\prime}$ and each pair of operations $i=J_{r}, j=J_{r^{\prime}}$, with $r<r^{\prime}$ and $m_{i}=m_{j}$, add an $\operatorname{arc}\left(v_{i}, v_{j}\right)$ with length $p_{i}+s_{i j}$ reflecting the setup between operations of a same job.

The set of disjunctive arcs $E$ is built by adding for each pair of operations $i, j \in I$ from distinct jobs using a same machine an $\operatorname{arc}\left(v_{i}, v_{j}\right)$ with length $p_{i}+s_{i j}$ and an arc $\left(v_{j}, v_{i}\right)$ with length $p_{j}+s_{j i}$. The pairs of disjunctive arcs $\left\{\left(v_{i}, v_{j}\right),\left(v_{j}, v_{i}\right)\right\}$ form the disjunctive sets of $\mathscr{E}$.

For ease of notation, let $\alpha_{J}=\alpha_{J_{|J|}}+p_{J_{|J|}}$ be the time at which job $J \in \mathscr{J}$ is finished. The following five objective functions are considered in the sequel of the paper: makespan $f^{\mathrm{m}}(\alpha)=\max _{J \in \mathscr{J}} \alpha_{J}$, total flow time $f^{\mathrm{f}}(\alpha)=\sum_{J \in \mathscr{J}} \alpha_{J}$, total squared flow time $f^{\mathrm{sf}}(\alpha)=\sum_{J \in \mathscr{J}} \alpha_{J}^{2}$, total tardiness $f^{\mathrm{t}}(\alpha)=\sum_{J \in \mathscr{J}} \max \left\{0, \alpha_{J}-s_{J}^{\mathrm{d}}\right\}$, and total weighted tardiness $f^{\mathrm{wt}}(\alpha)=\sum_{J \in \mathscr{J}} w_{J} \cdot \max \left\{0, \alpha_{J}-s_{J}^{\mathrm{d}}\right\}$ where $w_{J}$ is a given nonnegative weight of job $J$.

A small example of a JSS instance is illustrated in Fig. 2. It consists of ten operations $I=\{1, \ldots, 10\}$ and four jobs $J=(1,2,3), K=(4,5), L=(6,7)$, and $N=(8,9,10)$. The processing data can be directly read in the solution depicted in the Gantt chart of Fig. 2. For example, operation 2 is executed on machine $M_{4}$ and has duration 2. The setup times between the operations on machine $M_{1}$ are: $s_{1,5}=2$, $s_{1,8}=1, s_{5,1}=2, s_{5,8}=1, s_{8,1}=1, s_{8,5}=3$, and all other setup times are 0 . The release time of job $L$ is 5 and all other release times are 0 . The due date is 20,30,20, and 20 for job $J, K, L$, and $N$, respectively. Finally, the weight of job $J$ is 3 and all other job weights are 1.

\subsubsection{The blocking job shop with transfer times, setup times, and a regular objective}

The blocking job shop problem with transfer times, setup times, and a regular objective (BJS) is a version of the JSS introduced above, characterized by the absence of buffer capacity. This additional feature implies that, after completing an operation, a job waits on its current machine, thus blocking it, until it is transferred to its next machine. While transferring a job, both involved machines are simultaneously occupied. Each operation of a job is then best described by the four consecutive steps: (i) a take-over step in which the job is taken over from its previous machine, (ii) a processing step, (iii) a possible waiting step on its current machine, (iv) a hand-over step in which the job is transferred to its next machine. For the first operation of a job, the take-over step is more appropriately called loading step, and similarly, for the last operation of a job, the hand-over step is called unloading step. We refer to (Gröflin and Klinkert, 2009; Gröflin et al, 2011) for a more detailed description of the BJS. In the three field notation, the BJS is specified by $J \mid$ blocking, $r_{j}, s_{i j} \mid$ reg. 

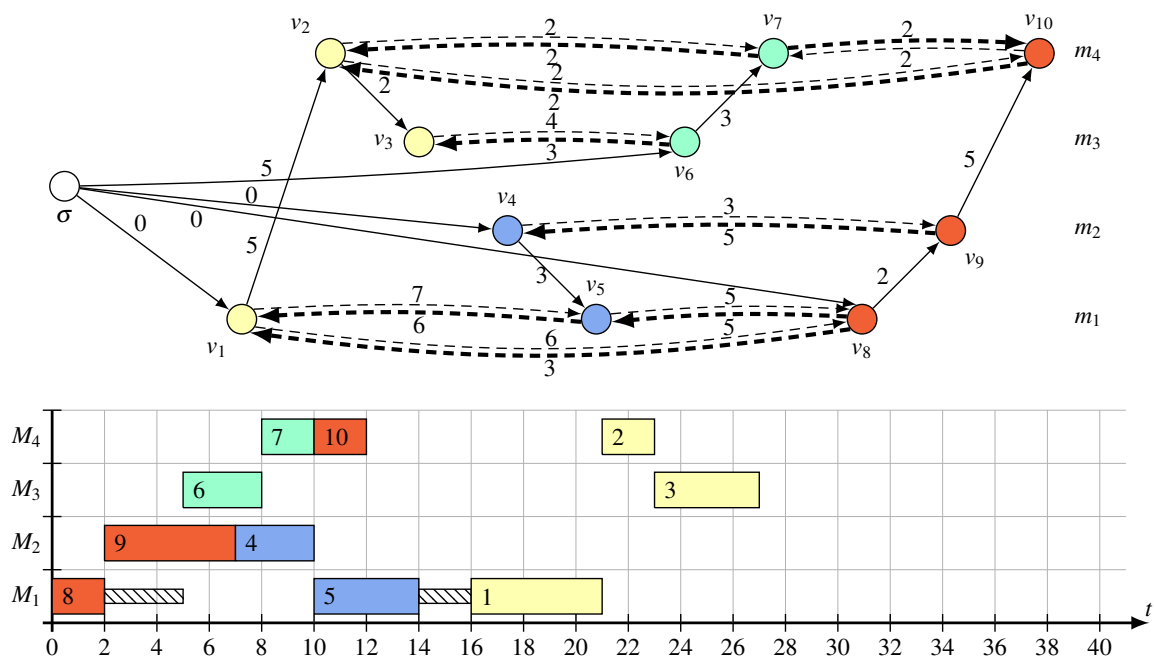

Fig. 2: A JSS instance with four jobs and four machines. (top) Its associated disjunctive graph $G$. Conjunctive and disjunctive arcs are depicted by solid and dashed lines, respectively. (bottom) A Gantt chart of the earliest time schedule $\alpha(S)$ of selection $S$ consisting of the bold, dotted disjunctive arcs of $G$. The values of the five introduced objectives are: $f^{\mathrm{m}}(\alpha(S))=27, f^{\mathrm{f}}(\alpha(S))=63, f^{\mathrm{sf}}(\alpha(S))=1169, f^{\mathrm{t}}(\alpha(S))=7$, and $f^{\mathrm{wt}}(\alpha(S))=21$. In the Gantt chart, each operation is depicted by one bar, and the narrow, hatched bars display setups.

In order to formally define the BJS problem, the following additional notation and data is used. Let $t_{i j}$ be the time needed to transfer the job of operation $i$ from machine $m_{i}$ to its next machine $m_{j}$, where $i$ and $j$ are consecutive operations in a job. Also, let $t_{J}^{1}$ and $t_{J}^{\mathrm{u}}$ be the time needed to load job $J \in \mathscr{J}^{\prime}$ on its first machine and unload it from its last machine, respectively. Denote by $I^{\text {first }} \subseteq I$ and $I^{\text {last }} \subseteq I$ the subset of all first and last operations of jobs, respectively.

The BJS can then be described as a CJS-R problem in graph $G=(V, A, E, \mathscr{E}, d)$ as follows (see also the example below, which is illustrated in Fig. 3). For each operation $i \in I$, add four nodes to $V: v_{i}^{1}$ (start of take-over), $v_{i}^{2}$ (end of take-over), $v_{i}^{3}$ (start of hand-over), and $v_{i}^{4}$ (end of hand-over). Note that the end of the take-over step is also the start of the processing step. For each job $J^{\prime} \in \mathscr{J}^{\prime}$, introduce a job $J$ in $\mathscr{J}$ containing all nodes of the operations belonging to $J^{\prime}$.

The set of conjunctive $\operatorname{arcs} A$ is built as follows:

- For each job $J \in \mathscr{J}^{\prime}$, add an arc $\left(\sigma, v_{J_{1}}^{1}\right)$ with length $s_{J}^{\mathrm{r}}$ reflecting the release time of job $J$.

- For each operation $i \in I$, add three arcs: a take-over $\operatorname{arc}\left(v_{i}^{1}, v_{i}^{2}\right)$ with length $t_{J o b(i)}^{1}$ if $i \in I^{\text {first }}$ and 0 otherwise, a processing arc $\left(v_{i}^{2}, v_{i}^{3}\right)$ with length $p_{i}$, and a handover $\operatorname{arc}\left(v_{i}^{3}, v_{i}^{4}\right)$ with length $t_{J o b(i)}^{\mathrm{u}}$ if $i \in I^{\text {last }}$ and 0 otherwise.

- For any two consecutive operations $i, j$ of some job $J \in \mathscr{J}^{\prime}$, add two pairs of arcs $\left(v_{i}^{3}, v_{j}^{1}\right),\left(v_{j}^{1}, v_{i}^{3}\right)$ and $\left(v_{i}^{4}, v_{j}^{2}\right),\left(v_{j}^{2}, v_{i}^{4}\right)$ of length 0 synchronizing the start and end of the hand-over step of $i$ with the start and end of the take-over step of $j$, and add an $\operatorname{arc}\left(v_{i}^{3}, v_{j}^{2}\right)$ of length $t_{i j}$ reflecting the transfer of the job. 

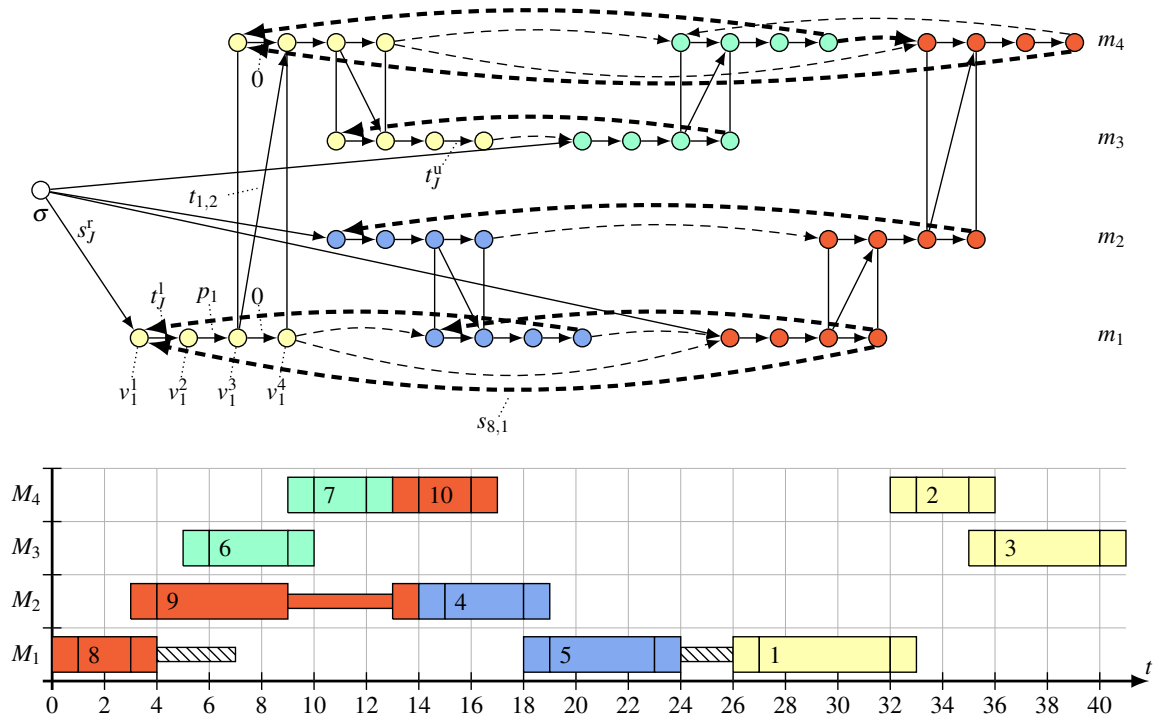

Fig. 3: The BJS example. (top) Its associated disjunctive graph $G$. (bottom) A Gantt chart of the earliest time schedule $\alpha(S)$ of selection $S$ consisting of the bold, dotted disjunctive arcs of $G$. Its objective values are: $f^{\mathrm{m}}(\alpha(S))=41, f^{\mathrm{f}}(\alpha(S))=95, f^{\mathrm{sf}}(\alpha(S))=2715, f^{\mathrm{t}}(\alpha(S))=21$, and $f^{\mathrm{wt}}(\alpha(S))=63$. In the Gantt chart, the take-over, processing, and hand-over steps are depicted by thick bars. The narrow bars stand for the setups (hatched) and the waiting steps (solid).

- For each job $J \in \mathscr{J}^{\prime}$ and each pair of operations $i=J_{r}, j=J_{r^{\prime}}$ with $r<r^{\prime}$ and $m_{i}=m_{j}$, add an arc $\left(v_{i}^{4}, v_{j}^{1}\right)$ with length $s_{i j}$ reflecting the setup between operations of a same job.

The set of disjunctive arcs $E$ is built by adding for each pair of operations $i, j \in I$ from distinct jobs using a same machine an $\operatorname{arc}\left(v_{i}^{4}, v_{j}^{1}\right)$ with length $s_{i j}$ and an arc $\left(v_{j}^{4}, v_{i}^{1}\right)$ with length $s_{j i}$. The pairs of disjunctive $\operatorname{arcs}\left\{\left(v_{i}^{4}, v_{j}^{1}\right),\left(v_{j}^{4}, v_{i}^{1}\right)\right\}$ form the disjunctive sets of $\mathscr{E}$.

We consider the same five regular objective functions as in the JSS. Note that the time $\alpha_{J}$ at which a job $J \in \mathscr{J}$ is finished is $\alpha_{J}=\alpha_{v_{i}^{4}}$ with $i=J_{|J|}$.

We reconsider the JSS example introduced in the previous section as BJS instance. Let the durations of all take-over, hand-over, loading, and unloading steps be 1. Fig. 3 depicts the disjunctive graph $G$ of this example and a Gantt chart of a feasible schedule.

Note that the given formulation of the BJS is not the most compact form. It is for instance easy to see that nodes occurring at the same time can be represented by a single node. In our view, the given form is more readable than, for example, the so-called alternative graph formulation presented in (Mascis and Pacciarelli, 2002). 


\section{A special subproblem: the job insertion problem}

We first describe and formulate the job insertion problem and then present some structural properties. These are based on results of Gröflin and Klinkert (2007) and are specialized to the problem under study. Proofs that are similar as those in the referenced work are provided in the appendix.

\subsection{The job insertion problem}

Informally, job insertion can be thought of as the following problem. Given a job and a feasible selection of all other jobs, insert the job in such a way that the resulting schedule is feasible, and, if an optimal insertion is sought, find a feasible insertion with minimum objective value.

Specifically, given a CJS-R problem $(V, \mathscr{J}, A, E, \mathscr{E}, d, f)$ and a job $J \in \mathscr{J}$, a feasible selection of all other jobs is specified in disjunctive graph $G=(V, A, E, \mathscr{E}, d)$ by a selection $R$ that is positive acyclic and "complete", i.e., $D \cap R \neq \emptyset$ holds for each pair $D=\left\{(v, w),\left(v^{\prime}, w^{\prime}\right)\right\} \in \mathscr{E}$ with $\{v, w\} \cap V_{J}=\emptyset$. We are interested in finding a feasible selection $S=T \cup R$, where $T$ is more appropriately called a feasible insertion of job $J$.

We study the job insertion problem in the insertion graph $G^{J}=\left(V, A^{J}, E^{J}, \mathscr{E}^{J}, d\right)$ of job $J$ derived from $G$ as follows. Set $A^{J}$ of conjunctive arcs is obtained by adding to $A$ the set $R$, and set $E^{J}$ is obtained by deleting from $E$ all disjunctive arcs that are not incident to job $J$. Formally, $A^{J}=A \cup R, E^{J}=E \cap \delta\left(V_{J}\right)$, and $\mathscr{E}^{J}=\left\{D \in \mathscr{E}: D \subseteq E^{J}\right\}$. Note that by (8), if $e \in E^{J}$ then also $\bar{e} \in E^{J}$. Clearly, $T \subseteq E^{J}$ is a (positive acyclic, complete, feasible) insertion in $G^{J}$ if and only if $S=T \cup R$ is a (positive acyclic, complete, feasible) selection in $G$.

\subsection{Structural properties of job insertion}

Given is a job insertion problem of job $J$ in its insertion graph $G^{J}=\left(V, A^{J}, E^{J}, \mathscr{E} J, d\right)$. We examine cycles in graph $\left(V, A^{J} \cup E^{J}, d\right)$. The fictive node $\sigma$ is never part of a cycle as $\left(V, A^{J} \cup E, d\right)$ contains no arcs entering $\sigma$. By (6), all conjunctive arcs incident to job $J$ are of the type $(\sigma, v)$, hence any cycle $Z$ in $\left(V, A^{J} \cup E^{J}, d\right)$ enters job $J$ through disjunctive $\operatorname{arcs}$ in $\delta^{-}\left(V_{J}\right)$ and leaves $J$ through disjunctive $\operatorname{arcs}$ in $\delta^{+}\left(V_{J}\right)$, and the number of times $Z$ enters and leaves $J$ is the same, i.e., $\left|Z \cap \delta^{-}\left(V_{J}\right)\right|=\left|Z \cap \delta^{+}\left(V_{J}\right)\right|=$ $k$ for some $k \geq 1$. Number $k$ can be seen as the number of times $Z$ visits job $J$.

Considering any positive cycle $Z$ in $\left(V, A^{J} \cup E^{J}, d\right)$, it is easy to see that $Z$ must visit $J$ at least once. Indeed, the conjunctive part $\left(V, A^{J}, d\right)$ of $G^{J}$ contains no positive cycles since $R$ is a positive acyclic selection in $G$ and $A^{J}=A \cup R$. We now capture all positive cycles visiting job $J$ exactly once in a so-called conflict graph.

Definition 2 The conflict graph of job insertion graph $G^{J}=\left(V, A^{J}, E^{J}, \mathscr{E}^{J}, d\right)$ is the undirected graph $H=\left(E^{J}, U\right)$ where for any two distinct disjunctive arcs $e, f \in E^{J}$, edge $\{e, f\} \in U$ if insertion $\{e, f\}$ is positive cyclic. 

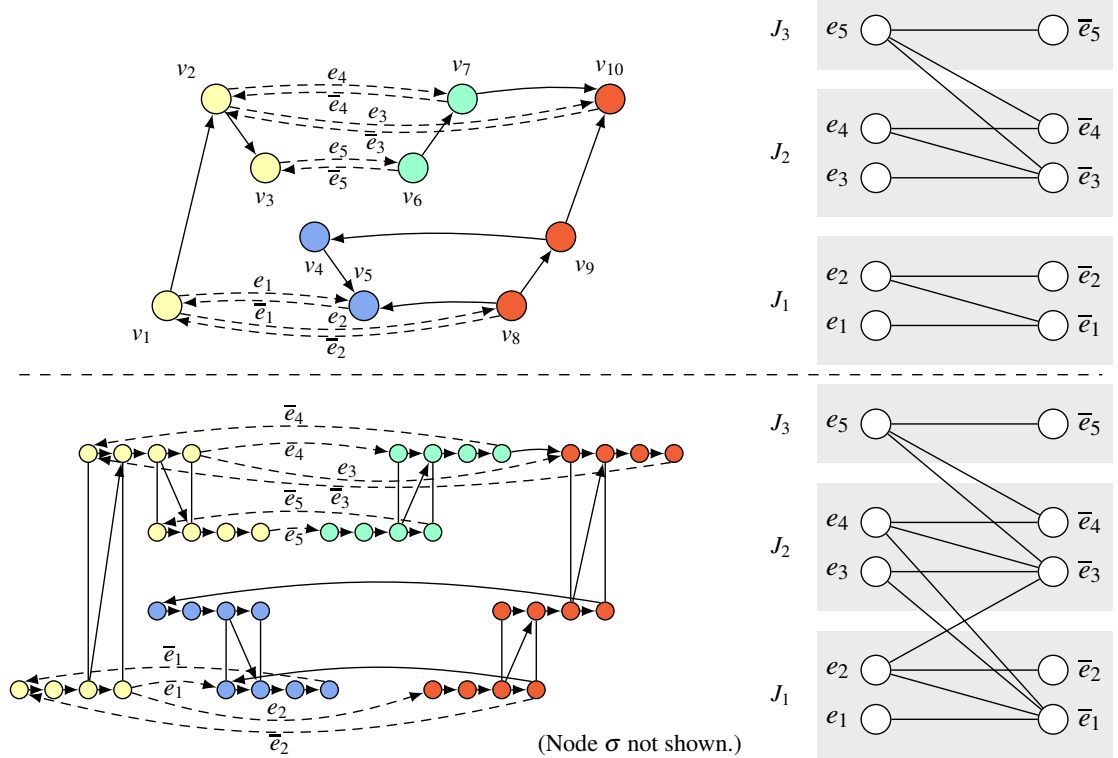

Fig. 4: The job insertion graph and its associated conflict graph for the JSS example (above) and the BJS example (below). In both cases, the job insertion problem of job $J$ (consisting of the operations 1,2 , and 3 ) is considered. The feasible selections of all other jobs are taken from Fig. 2 and 3.

Fig. 4 depicts the job insertion graph and its associated conflict graph for the JSS and BJS examples.

Based on the previous discussion and by (9), it is easy to see that the conflict graph $H$ has the following structural properties.

Proposition 1 Let $G^{J}=\left(V, A^{J}, E^{J}, \mathscr{E}^{J}, d\right)$ be a job insertion graph and $H=\left(E^{J}, U\right)$ its associated conflict graph.

(i) $\{e, \bar{e}\} \in H$ for each disjunctive arc pair $\{e, \bar{e}\} \in \mathscr{E}^{J}$.

(ii) The conflict graph is bipartite with partitions $E^{J-}=E^{J} \cap \delta^{-}\left(V_{J}\right)$ and $E^{J+}=$ $E^{J} \cap \delta^{+}\left(V_{J}\right)$.

We can establish the following relations between the feasible insertions in $G^{J}$ and the stable sets in $H$.

Proposition 2 Let $G^{J}=\left(V, A^{J}, E^{J}, \mathscr{E}^{J}, d\right)$ be a job insertion graph and $H=\left(E^{J}, U\right)$ its associated conflict graph.

(i) Any feasible insertion in $G^{J}$ corresponds to a stable set in conflict graph $H$ of size $\left|E^{J}\right| / 2$.

(ii) Any stable set in $H$ of size $\left|E^{J}\right| / 2$ corresponds to a complete insertion.

Proof See appendix.

Unfortunately, an insertion $T$ that corresponds to a stable sets of size $\left|E^{J}\right| / 2$ can be positive cyclic as cycles visiting job $J$ more than once may be present in graph 
$\left(V, A^{J} \cup T, d\right)$. We introduce a structural property of the job insertion graph preventing the existence of these cycles. It is called the short cycle property (SCP) and was first introduced in (Gröflin and Klinkert, 2007) for general disjunctive graphs.

Definition 3 A job insertion graph $G^{J}$ has the SCP if for any positive cycle with arc set $Z^{\prime}$ in $\left(V, A^{J} \cup E^{J}, d\right)$, there exists a short positive cycle $Z$ in $\left(V, A^{J} \cup E^{J}, d\right)$ with $Z \cap E^{J} \subseteq Z^{\prime} \cap E^{J}$ and $\left|Z \cap E^{J}\right|=2$.

In other words, a job insertion graph has the SCP if for any positive cycle $Z^{\prime}$ visiting $J$ more than once, there exists a positive cycle $Z$ visiting $J$ exactly once entering and leaving $J$ through disjunctive arcs present in $Z^{\prime}$.

We say that a CJS-R problem has the SCP for job insertion if $G^{J}$ has the SCP for all jobs $J \in \mathscr{J}$ and all feasible selections $R$ of the other jobs.

Theorem 1 Let $G^{J}$ be a job insertion graph with the SCP. There is a one-to-one correspondence between the feasible insertions in $G^{J}$ and the stable sets $T$ in conflict graph $H$ with $|T|=\left|E^{J}\right| / 2$.

Proof See appendix.

Hence, if a CJS-R problem has the SCP for job insertion, a nice characterization of all feasible insertions as stable sets in an associated bipartite conflict graph is at hand for all job insertion subproblems.

\subsection{The short cycle property in specific job shop problems}

Unfortunately not all, but a large class of CJS-R problems has the SCP for job insertion. We show here that the JSS and BJS problems have the SCP for job insertion. We give a similar proof as in Lemma 8 of Gröflin and Klinkert (2007) and refer the reader to this work for general structural results.

Proposition 3 Let $G^{J}=\left(V, A^{J}, E^{J}, \mathscr{E} J, d\right)$ be a job insertion graph of a JSS instance for some job $J \in \mathscr{J}$. Then $G^{J}$ has the SCP.

Proof Given any positive cycle $Z^{\prime}$, let $Z$ be a "shortest" positive cycle in graph $\left(V, A^{J} \cup E^{J}, d\right)$ with $Z \cap E^{J} \subseteq Z^{\prime} \cap E^{J}$ where the length is measured by the number of disjunctive arcs $\left|Z \cap E^{J}\right|$. If $\left|Z \cap E^{J}\right|=2$, we are done.

Assume $\left|Z \cap E^{J}\right|>2$, and say $Z$ visits job $J k=\left|Z \cap E^{J}\right| / 2$ times. Describe $Z$ by the concatenation $Z=\left(e_{1}, P_{1}, e_{1}^{\prime}, Q_{1}, e_{2}, P_{2}, e_{2}^{\prime}, Q_{2}, \ldots, e_{k}, P_{k}, e_{k}^{\prime}, Q_{k}\right)$ where the $e_{i}^{\prime}$ 's and $e_{i}^{\prime}$ 's are disjunctive arcs entering and leaving $J$, respectively, the $P_{i}$ 's are paths in $\left(V, \gamma\left(V_{J}\right), d\right)$ and the $Q_{i}$ 's are paths in $\left(V, \gamma\left(V \backslash V_{J}\right), d\right)$.

The following two simple properties of the subgraph $\left(V, \delta\left(A^{J}\right), d\right)$ are used. For any pairs of distinct operations $J_{r}, J_{s}$ : i) If $r<s$, there exists a path from node $v_{r}$ to node $v_{s}$ and such as path is of positive length. Indeed, it must contain arc $\left(v_{r}, v_{r+1}\right)$, which is of length $p_{J_{r}}>0$, and no arc is of negative length. ii) If $r>s$, it is easy to see that there exists no path from $v_{r}$ to $v_{s}$.

Consider $P_{1}$ and $P_{2}$ of cycle $Z$. $P_{1}$ starts at node $v_{J_{r}}$ of some operation $J_{r}$ and ends at node $v_{J_{s}}$ of some $J_{s}$. Similarly, $P_{2}$ starts at some $v_{J_{r^{\prime}}}$ and ends at some $v_{J_{s^{\prime}}}$. By ii), 
$r \leq s$ and $r^{\prime} \leq s^{\prime}$ hold, and as $Z$ is a cycle, $r \neq r^{\prime}$ (a cycle does not enter the same node more than once).

Consider the following two cases. a) If $r<r^{\prime}$, let concatenation $W=\left(e_{1}, P^{*}, e_{2}^{\prime}\right.$, $\left.Q_{2}, \ldots, e_{k}, P_{k}, e_{k}^{\prime}, Q_{k}\right)$ where $P^{*}$ is a path from node $v_{r}$ to node $v_{s^{\prime}}$ in $\left(V, \gamma\left(V_{J}\right), d\right)$. By i), path $P^{*}$ exists and is of positive length as $r<r^{\prime}$ and $r^{\prime} \leq s^{\prime}$ implies $r<s^{\prime}$. Then $W$ is of positive length as no arc in $G^{J}$ is of negative length arcs. Clearly, $W$ is a closed walk visiting $J$ less than $k$ times. Therefore, $W$ can be decomposed into cycles (cycle decomposition of integer circulations) where each cycle visits $J$ at least once and less than $k$ times. As $W$ is of positive length, one of these cycles, say $Z^{\prime \prime}$, is also of positive length.

b) If $r>r^{\prime}$ then consider the cycle $Z^{\prime \prime}=\left(e_{2}, P^{*}, e_{1}^{\prime}, Q_{1}\right)$, where $P^{*}$ is a path from node $v_{r^{\prime}}$ to node $v_{s}$ in $\left(V, \gamma\left(V_{J}\right), d\right)$. By i), path $P^{*}$ exists and is of positive length as $r^{\prime}<r$ and $r \leq s$ implies $r^{\prime}<s$. Then $Z^{\prime \prime}$ is of positive length.

In both cases, $Z^{\prime \prime}$ is a positive cycle with $Z^{\prime \prime} \cap E^{J} \subseteq Z^{\prime} \cap E^{J}$ visiting $J$ less than $k$ times, contradicting the choice of $Z$ as being the shortest.

Proposition 4 Let $G^{J}=\left(V, A^{J}, E^{J}, \mathscr{E}^{J}, d\right)$ be a job insertion graph of a BJS instance for some job $J \in \mathscr{J}$. Then $G^{J}$ has the $S C P$.

Proof The proof is similar to the proof given above. The only differences are the properties of subgraph $\left(V_{J}, \delta\left(A^{J}\right), d\right)$ we use, namely: i) For any pair of operations $J_{r}, J_{s}$ with $r \leq s$, there exists a path from node $v_{r}^{1}$ (of operation $J_{r}$ ) to node $v_{s}^{4}$ (of $J_{s}$ ) and it is of positive length as it must contain the processing arc $\left(v_{r}^{2}, v_{r}^{3}\right)$ and no arc is of negative length. And ii) for any pair of operations $J_{r}, J_{s}$ with $r>s+1$, there exists no path from node $v_{r}^{1}$ (of operation $J_{r}$ ) to node $v_{s}^{4}$ (of $J_{s}$ ).

Then, as above, take a "shortest" positive cycle and, and, if it visits $J$ more than once, say $k$ times, construct a positive cycle $Z^{\prime \prime}$ visiting $J$ less than $k$ times.

Hence, independent of the choice of the job $J$ and the feasible selection $R$ for all other jobs, $G^{J}$ has the SCP in JSS and BJS problems. Therefore, JSS and BJS problems have the SCP for job insertion.

\section{A neighborhood for the CJS-R}

In this section, we develop a neighborhood that is applicable to all CJS-R problems possessing the SCP for job insertion.

\subsection{A neighbor generation scheme}

Given is a CJS-R problem $(V, \mathscr{J}, A, E, \mathscr{E}, d, f)$ with the SCP for job insertion. Let $S$ be some feasible selection. We are interested in extracting some job $J \in \mathscr{J}$ and reinserting $J$ into a position that is "close" to its position described by selection $S$. For this purpose, we consider the job insertion graph $G^{J}=\left(V, A^{J}, E^{J}, \mathscr{E}^{J}, d\right)$, where the feasible selection of all other jobs $R$ is taken from $S$, i.e. $R=S \cap\left(E \backslash E^{J}\right)$. We choose a disjunctive arc $g \in E^{J} \backslash S$ that is forced to be in the neighbor insertion. Then 
we ask to construct a feasible insertion $T_{g}$ with $g \in T$ that is "close" to the current insertion $T_{S}=S \cap E^{J}$, i.e., we seek to find a feasible insertion $T_{g}$ maximizing $\left|T_{g} \cap T_{S}\right|$. The corresponding neighbor selection $S_{g}$ is then obtained by $S_{g}=T_{g} \cup R$.

Consider the neighbor construction in the conflict graph $H=\left(E^{J}, U\right)$ associated to $G^{J}$. By Theorem $1, T_{S}$ is stable in $H$ with size $\left|E^{J}\right| / 2$, and any feasible insertion $T_{g}$ is stable in $H$ with size $\left|E^{J}\right| / 2$. We now sketch how to iteratively construct $T_{g}$. The procedure is based on the following observation. For any $f \in T_{g}$, if there exists an edge $\{f, e\} \in U$, then $e$ cannot be picked, hence its mate $\bar{e}$ must be part of any feasible insertion containing $f$. We say $f$ implies $\bar{e}$. Hence, start with $T_{g}=\{g\}$. For each $f \in T_{g}$ and $\{f, e\} \in U$ with $\bar{e} \notin T_{g}$, add $\bar{e}$ to $T_{g}$. Repeat this step until $T_{g}$ is "closed", i.e., for each $f \in T_{g}$ there exists no $\{f, e\} \in U$ with $\bar{e} \notin T_{g}$. Finally, for each "uncovered" pair $D \in \mathscr{E}$, i.e., $D \cap T_{g}=\emptyset$, add $e \in D \cap T_{S}$ from the current insertion $T_{S}$ to $T_{g}$.

We formalize this idea by introducing a closure operator and then prove feasibility of the constructed neighbor insertion.

Definition 4 For any $e, f \in E^{J}$ in conflict graph $H=\left(E^{J}, U\right)$, let $e \rightarrow f$ if $\{e, \bar{f}\} \in$ $U$. A sequence $P=\left(e_{0}, e_{1}, \ldots, e_{n}\right), n \geq 0$, of distinct nodes in $H=\left(E^{J}, U\right)$ is an alternating path from $e_{0}$ to $e_{n}$ if $e_{i} \rightarrow e_{i+1}$ for all $0 \leq i<n$. For any two nodes $e, f \in$ $E^{J}$, write $e \rightsquigarrow f$ if there exists an alternating path from $e$ to $f$ in $H$.

Definition 5 For any $Q \in E^{J}$, the closure of $Q$ is the set

$$
\Phi(Q)=\left\{g \in E^{J}: e \rightsquigarrow g \text { for some } e \in Q\right\} .
$$

$Q \subseteq E^{J}$ is said to be closed if $Q=\Phi(Q)$.

Observe that $e \rightsquigarrow e$ holds for all $e \in E^{J}$ as $\{e, \bar{e}\} \in U$ by Proposition 1(i). Moreover, $\Phi(Q)$ can be rewritten as $\Phi(Q)=\bigcup_{e \in Q} \Phi(\{e\})$. Then, it is easy to see that $\Phi$ is a well-defined closure operator as: i) $Q \subseteq \Phi(Q)$, ii) $\Phi(\Phi(Q))=\Phi(Q)$, and iii) $Q \subseteq R$ implies $\Phi(Q) \subseteq \Phi(R)$.

For any subset of disjunctive $\operatorname{arcs} T \in E^{J}$, let the span $[T]$ of $T$ contain all arcs of $T$ together with all their mates, formally, $[T]=\left\{e \in E^{J}:\{e, \bar{e}\} \cap T \neq \emptyset\right\}$.

Theorem 2 Given any job insertion graph $G^{J}=\left(V, A^{J}, E^{J}, \mathscr{E}^{J}, d\right)$ with the SCP and a disjunctive arc $g \in E^{J}$, insertion

$$
T_{g}=\Phi(\{g\}) \cup\left(T^{S} \backslash[\Phi(\{g\})]\right)
$$

is feasible.

Proof See appendix.

It is easy to see that the constructed neighbor $T_{g}$ is the "closest" to neighbor $T^{S}$, i.e. among all neighbor insertions $T$ containing $g$, it maximizes $\left|T_{g} \cap T_{S}\right|$.

Theorem 2 implies feasibility of the corresponding selection, stated as follows. 

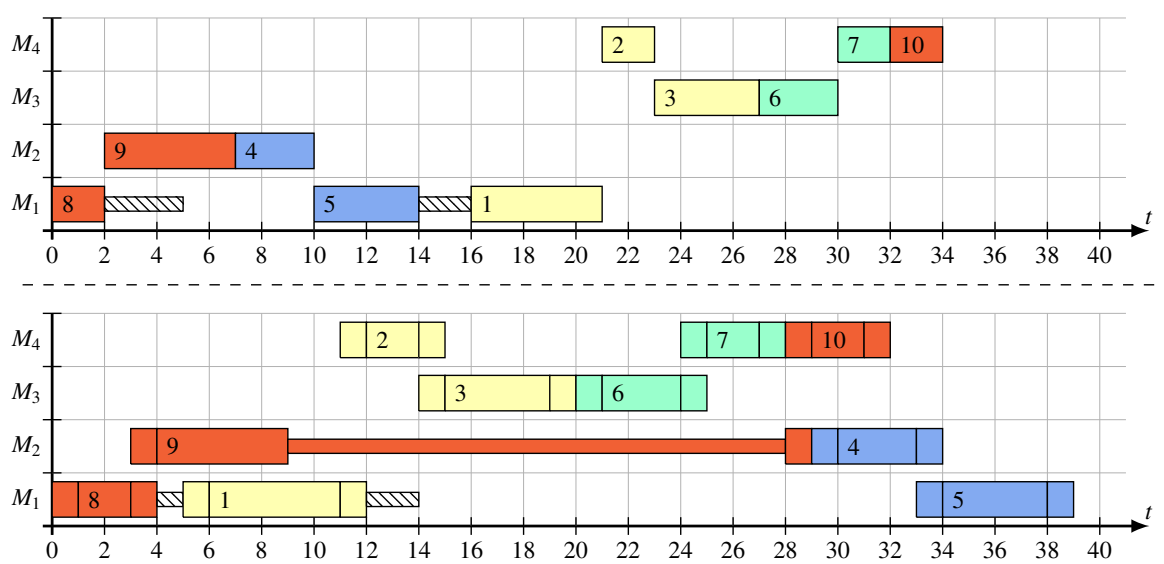

Fig. 5: The neighbor schedules obtained by extracting job $J$ (consisting of the operations 1, 2, and 3 ) from the schedule of Fig. 2 and 3 in the JSS (above) and BJS example (below), respectively, and reinserting this job by forcing operation 3 to be executed before operation 6 on machine $M_{3}$.

Corollary 1 Given a CJS-R problem $(V, \mathscr{J}, A, E, \mathscr{E}, d, f)$ with the SCP for job insertion, for any feasible selection $S$, job $J \in \mathscr{J}$, and disjunctive arc $g \in E^{J}$, the neighbor selection

$$
S_{g}^{J}=T_{g} \cup\left(S \backslash E^{J}\right)
$$

is feasible.

We illustrate the neighbor generation scheme in the JSS and BJS examples. Starting with the selections given in Fig. 2 and 3, we choose job $J$ to be extracted and reinserted, obtaining the job insertion graphs and conflict graphs depicted in Fig. 4. In both examples, the current insertion $T^{S}=\left\{\bar{e}_{1}, \bar{e}_{2}, \bar{e}_{3}, \bar{e}_{4}, \bar{e}_{5}\right\}$ places job $J$ after all other jobs. We force $e_{5}$ to be part of the neighbor insertion. In the JSS example, we obtain $\Phi\left(\left\{e_{5}\right\}\right)=\left\{e_{3}, e_{4}, e_{5}\right\}$ and $T^{S} \backslash[\Phi(\{g\})]=\left\{\bar{e}_{1}, \bar{e}_{2}\right\}$. Hence, the neighbor insertion is $T_{g}=\left\{\bar{e}_{1}, \bar{e}_{2}, e_{3}, e_{4}, e_{5}\right\}$. In the BJS example, we obtain $\Phi\left(\left\{e_{5}\right\}\right)=\left\{e_{1}, e_{3}, e_{4}, e_{5}\right\}$ and $T^{S} \backslash[\Phi(\{g\})]=\left\{\bar{e}_{2}\right\}$. Hence, the neighbor insertion is $T_{g}=\left\{e_{1}, \bar{e}_{2}, e_{3}, e_{4}, e_{5}\right\}$. The corresponding earliest time schedules are illustrated in Fig. 5.

Note that if an instance of the classical job shop scheduling problem is given and $\bar{g}$ is a critical arc incident to job $J$ in some feasible selection $S$, then $\Phi(\{g\})=\{g\}$, and the neighbor $S_{g}^{J}=\{g\} \cup(S \backslash\{\bar{g}\})$. Hence $S_{g}^{J}$ is obtained by replacing the critical $\operatorname{arc} \bar{g}$ by its mate $g$, which is typically called swapping a critical arc. In this sense, the proposed neighbor generation scheme can be seen as a generalization of swapping a critical arc.

\subsection{A job-insertion-based neighborhood}

Corollary 1 provides a general tool to derive a large set of neighbors for any feasible selection given the possible choices of the job and the "forced" disjunctive arc. In 
order to generate neighbors that are potentially improving the current solution, we use and slightly extend the standard concept of critical arcs as follows.

For any given selection $S$, an operation with latest completion time determines the makespan. With the makespan objective, we call such an operation contributing. In the disjunctive graph, a longest path from the dummy start to a node representing a contributing operation is called a critical path. The disjunctive arcs of a critical path are called critical arcs. With a general regular objective, usually not only an operation with the latest completion time contributes to the objective value, but some subset of (times of) operations determine the objective value for a given selection $S$. Consequently, we call all of these operations contributing. The definitions of critical paths and critical arcs are the same as in the makespan case.

Formally, this can be specified as follows. Given is some CJS-R problem $(V, \mathscr{J}$, $A, E, \mathscr{E}, d, f)$ with the SCP for job insertion and some feasible selection $S$. Let $V^{\text {contr }}$ be a (setwise) minimal subset of nodes in $V$ determining the objective value for selection $S$. For each node $v \in V$, let $P_{v}$ be the arc set of a longest path from $\sigma$ to $v$ in graph $(V, A \cup S, d)$. For each $v \in V^{\text {contr }}$, path $P_{v}$ is called a critical path. The set of critical $\operatorname{arcs} C_{S}$ of $S$ is given by $C_{S}=\left\{e \in S: e \in P_{v}\right.$ for some $\left.v \in V^{\text {contr }}\right\}$. A job $J \in \mathscr{J}$ is said to be critical if $v \in V_{J}$ or $w \in V_{J}$ for some $(v, w) \in C_{S}$.

In order to move to a better neighbor, we need to replace some arcs of $C_{S}$. Indeed, by construction of $C_{S}, \alpha\left(S^{\prime}\right) \geq \alpha(S)$ holds for any feasible selection $S^{\prime}$ with $C_{S} \subseteq S^{\prime}$, hence $f\left(\alpha\left(S^{\prime}\right)\right) \geq f(\alpha(S))$ as function $f$ is regular.

In our neighborhood, we build a neighbor selection $S_{g}^{J}$ by (12) for each critical job $J$ and each critical arc $\bar{g} \in C_{S} \cap \delta\left(V_{J}\right)$. The size of the neighborhood is $2\left|C_{S}\right|$ as two neighbors are built for each critical arc. We remark that this size can be related to the number of operations $|I|$ for the JSS and BJS problems. As the setup durations satisfy the weak triangle inequality, we can assume that disjunctive arcs between nonconsecutive operations on some machine are not part of longest paths in $(V, A \cup S, d)$. It is easy to see that the number of disjunctive arcs between consecutive operations on a machine is $|I|-|M|$, where $|M|$ is the number of machines. Then, $\left|C_{S}\right| \leq|I|$ holds, implying that the neighborhood is at most of size $2|I|$.

We illustrate the neighborhood in the BJS example. Consider selection $S$ that corresponds to the solution depicted in Fig. 5 (below). In Fig. 6, the graph $(V, A \cup S, d)$ of selection $S$ is depicted in the upper part, and longest paths from $\sigma$ to nodes $v_{3}^{4}, v_{5}^{4}$, $v_{7}^{4}, v_{10}^{4}$ are depicted in the lower part.

With the makespan objective, the set of contributing nodes is $V^{\text {contr }}=\left\{v_{5}^{4}\right\}$ as the completion time of operation 5 determines the makespan. Inspecting the longest path from $\sigma$ to $v_{5}^{4}$ reveals that the set of critical arcs is $C_{S}=\{\bar{a}, \bar{b}, \bar{c}, \bar{d}\}$. With a total flow time objective, all end nodes of the jobs contribute to the objective value, hence $V^{\text {contr }}=\left\{v_{3}^{4}, v_{5}^{4}, v_{7}^{4}, v_{10}^{4}\right\}$. The set of critical arcs is then $C_{S}=\{\bar{a}, \bar{b}, \bar{c}, \bar{d}\}$. By chance, both objectives lead to the same set of critical arcs. Hence, with both objectives, the following eight neighbors are built: $S_{a}^{N}, S_{a}^{J}, S_{b}^{J}, S_{b}^{L}, S_{c}^{L}, S_{c}^{N}, S_{d}^{N}, S_{d}^{K}$. Their earliest time schedules are illustrated in Fig. 7. It can be seen, that the two neighbors generated with the same forced arc result in the same selection for $\operatorname{arcs} a, b$, and $c$, while the two neighbors are different for arc $d$. 

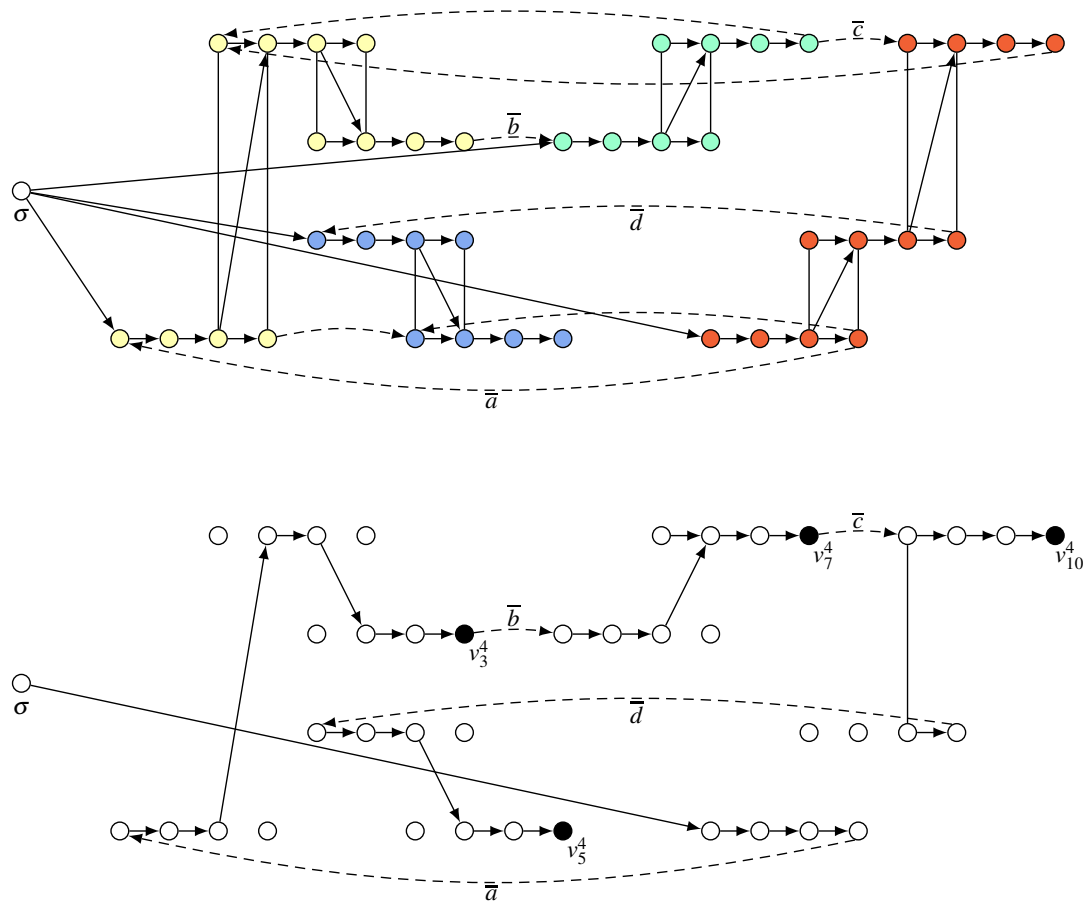

Fig. 6: (above) Graph $(V, A \cup S, d)$ of selection $S$ that corresponds to the BJS solution of Fig. 5 (below). The arcs of set $A$ and $S$ are depicted by solid and dashed lines, respectively. (below) Longest paths from $\sigma$ to nodes $v_{3}^{4}, v_{5}^{4}, v_{7}^{4}, v_{10}^{4}$ in graph $(V, A \cup S, d)$.

\section{Computational results}

In order to evaluate the performance of the proposed neighborhood, we casted it in a tabu search and conducted an extensive experimental study using well-known job shop scheduling problems. This section first sketches the tabu search, then describes the experimental setting, and finally discusses the obtained results.

\subsection{A tabu search}

In principle, the described neighborhood can be used in any local search scheme. We here apply it in a tabu search with some generic features that proved useful in various local search approaches for job shop scheduling problems. It is similar to the approach taken in (Gröflin et al, 2011; Bürgy, 2014) and will be called JILS (jobinsertion-based local search). We describe its main ingredients and refer the reader to Glover and Laguna (1997) for a comprehensive description of tabu search.

A tabu list $L$ is used for storing entries of the last $\max L$ iterations. Initially, list $L$ is empty. In an iteration, i.e., after moving from a selection $S$ to a neighbor $S_{g}^{J}$ by (12), $\operatorname{arc} \bar{g}$, which is the mate of the forced $\operatorname{arc} g$, is added to $L$ at first position and the oldest entry is deleted from $L$ if $|L|>\max L$. A neighbor $S^{\prime}$ is called tabu if $S^{\prime} \cap L \neq \emptyset$. 

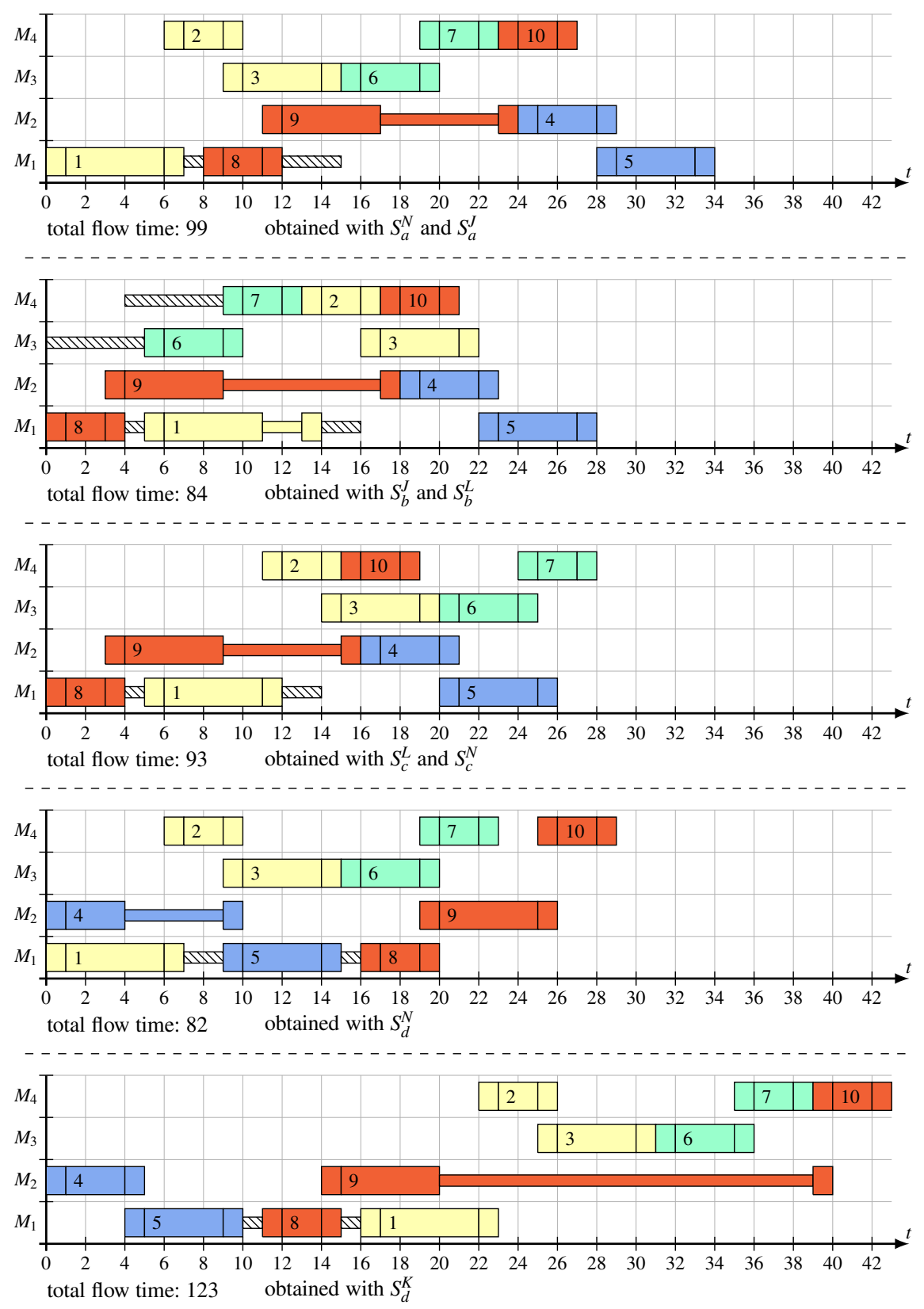

Fig. 7: An illustration of the neighborhood in the BJS example. For each attained earliest time schedule, we indicate the objective value and the neighbors leading to this schedule. 
Given a selection $S$, the choice of the move to be executed is based on the evaluation of the entire set of neighbors of $S$. First, the objective value $f\left(S^{\prime}\right)$ is computed for each neighbor $S^{\prime}$. Some of these values are "corrected" using the tabu list as follows. If a neighbor selection $S^{\prime}$ is tabu and $f\left(S^{\prime}\right)$ is not lower than the objective value of the best selection found so far, then a penalty value of $(\max L-k) \cdot B$ is added to the objective value, where $k$ is the position of the first entry in $L$ making the move tabu and $B$ is a large constant. $B$ should be chosen so that the corrected objective value of the tabu moves are higher than those of non-tabu moves. Finally, we select a neighbor with lowest (corrected) objective value.

In order to improve the search, we implemented the following two additional long term memory structures, which were also used by Nowicki and Smutnicki (1996).

As the tabu search does not prevent being trapped in long cycles, we use a list $C$ that stores the sequence of objective values obtained during the search. Cycles are detected by scanning $C$ for repeated subsequences. Specifically, the search is said to be cycling at iteration $k$ if there exists a period $\delta>\max L$ such that $C[k]=C[k-a \delta]$ for $a=1, \ldots, \max R$, where $C[j]$ is the objective value obtained in iteration $j, \max C$ reflects the maximum length of a cycle and $\max R$ is the number of repetitions we search for.

A list $E$ of so-called elite selections is maintained to diversify the search. Initially, $E$ is empty. A new selection $S$ is added to $E$ at first position if its objective value is lower than the value of the best selection found so far. If the search runs for a given number maxI of iterations without improving the best selection or if a selection has no neighbors or if a cycle is detected, then the current search path is terminated, list $C$ is cleared, and the search is resumed from the first selection of list $E$. For this purpose, an elite selection $S$ is stored together with its tabu list and the best (w.r.t. the objective value) $\max N$ neighbors that have not yet been directly visited from $S$. An elite selection is deleted from $E$ if its set of neighbors is empty.

In order to further diversify the search, we make use of the parallel computing capabilities by starting and running maxS independent search paths in parallel threads, each with its own initial selection, tabu list and list $C$. The $\max S$ search paths share list $E$ of elite selections and the best selection found so far.

\subsection{Initial selection}

Each of the $\max S$ initial selections is constructed as follows. First, we generate randomly a permutation of all jobs. According to this permutation, we then insert one by one a job into the current (partial) selection $S^{\text {part }}$. For the insertion of a job $J$ into $S^{\text {part }}$, we apply the job insertion procedure to generate a set of feasible selections $\mathscr{S}_{J}$ and choose the best selection among them. Specifically, a first selection $S_{J}$ is obtained by inserting $J$ after all other jobs, i.e. $S_{J}=S^{\text {part }} \cup E^{J-}$. Store $S_{J}$ in $\mathscr{S}_{J}$. While $E^{J-} \cap S_{J} \neq \emptyset$, execute the following three steps:

1. Determine a critical arc $f$ in $S_{J} \cap E^{J-}$.

2. If no such $f$ exists stop, else build neighbor selection $S_{\bar{f}}^{J}=T_{\bar{f}} \cup\left(S \backslash E^{J}\right)$ according to (12).

3. Store $S_{\bar{f}}^{J}$ in $\mathscr{S}_{J}$ and set $S_{J}$ to $S_{\bar{f}}^{J}$. 
Finally, among all selections in set $\mathscr{S}_{J}$, choose selection $S$ with lowest objective value and update $S^{\text {part }}$ to $S$.

We illustrate this procedure in the BJS example with job permutation $(J, K, L, N)$ (see Fig. 8). Job $J$ (operations 1, 2, and 3) has only one possible insertion as it is the first job in the permutation, see subfigure 1). In 2), job $K$ (op. 4 and 5) is placed after job $J$. The next insertion for $K$ is generated by forcing operation 5 to be moved before operation 1 as arc $\left(v_{1}^{4}, v_{5}^{1}\right)$ is critical, obtaining insertion 3$)$. This is the last insertion considered for job $K$, and we choose the insertion depicted in 2) for $K$ as it has a lower objective value than 3). In 4), job $L$ (op. 6 and 7 ) is placed after all other jobs. As arc $\left(v_{3}^{4}, v_{6}^{1}\right)$ is critical, we force operation 6 to be moved before operation 3 . Insertion 5) is obtained, which is the last insertion considered for job $L$. We choose the insertion depicted in 4) as it is better than 5). In 6), job $N$ (op. 8, 9, and 10) is placed after all other jobs. As arc $\left(v_{5}^{4}, v_{8}^{1}\right)$ is critical, we force operation 8 to be moved before operation 5. Insertion 7) is obtained. In this insertion, arc $\left(v_{7}^{4}, v_{10}^{1}\right)$ is critical, and we force operation 10 to be moved before operation 7 . Insertion 8 ) is obtained, in which $\operatorname{arc}\left(v_{1}^{4}, v_{8}^{1}\right)$ is critical. We force operation 8 to be moved before operation 1 and obtain insertion 9). In this insertion, $\operatorname{arc}\left(v_{2}^{4}, v_{10}^{1}\right)$ is critical, and we force operation 10 to be moved before operation 2. Thus, we obtain insertion 10). Among the insertions depicted in 6) to 10), insertion 6) is the best, hence we start the tabu search with its corresponding selection.

\subsection{Experimental setting}

JILS was implemented in Java and run on a PC with 3.3. GHz Intel Core i5-4590 processor (4 threads) and 16 GB memory.

Extensive tests were performed to evaluate JILS using the job shop problems introduced in Sect. 2.3.1 and 2.3.2 with various regular objectives. Specifically, we considered the job shop without setup times (JS), the job shop with sequence-dependent setup times (JSS), and the blocking job shop (BJS) with the five objective functions introduced in the examples: makespan, total flow time, total squared flow time, total tardiness, and total weighted tardiness.

We used standard benchmark instances from the literature: for the JS, la01-40 introduced by Lawrence (1984), orb01-010 by Applegate and Cook (1991), and ta0150 by Taillard (1994); for the JSS, t2-ps $X Y, X Y \in\{01, \ldots, 15\}$ by Brucker and Thiele (1996), t2-la $X Y$ sdst, $X Y \in\{21,24,25,27,29,38,40\}$, and t2-abzZsdst, $Z \in\{7,8,9\}$, by Vela et al (2010); and for the BJS, la01-40 by Lawrence (1984) and we set all transfer and setup times to 0 (obtaining so-called with-swap BJS instances).

In all problem types and instances, the release times are set to 0 . For the three tardiness objectives, the due date $s_{J}^{\mathrm{d}}$ of each job $J \in \mathscr{J}$ is set according to the rule introduced by Eilon and Hodgson (1967): $s_{J}^{\mathrm{d}}=\left\lfloor f * \sum_{i \in J} p_{i}\right\rfloor$ where $f$ is referred to as the due date tightness factor. $f$ is set to 1.3 for all JS instances, 1.6 for all JSS instances, and 1.8 for all BJS instances.

The input parameter settings of JILS were carefully analyzed in preliminary tests and set as follows: $\max C=4, \max I=50000, \max N=10, \max S=8$, and the tabu list size $\max L$ is 10 for all instances with the makespan objective and 16 for all other 


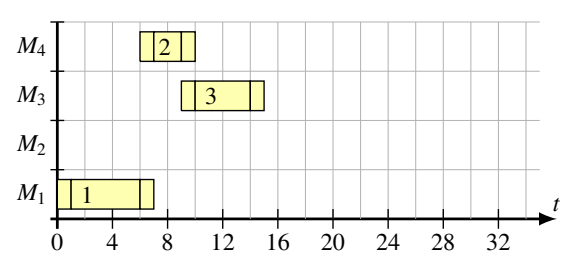

1) total flow time: 15

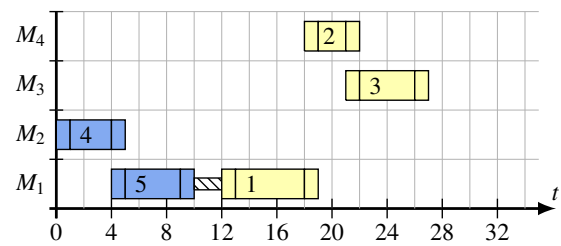

3) total flow time: 37

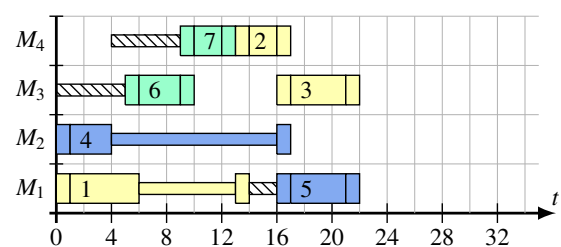

5) total flow time: 57

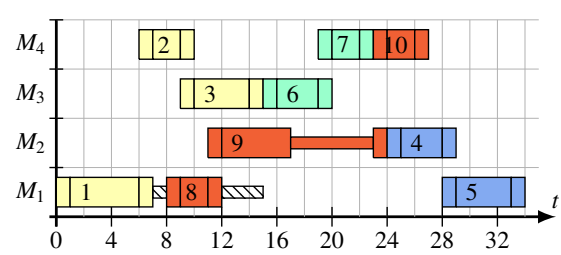

7) total flow time: 99

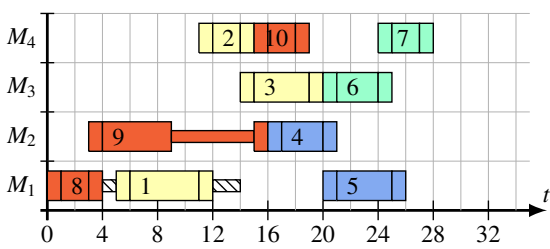

9) total flow time: 93

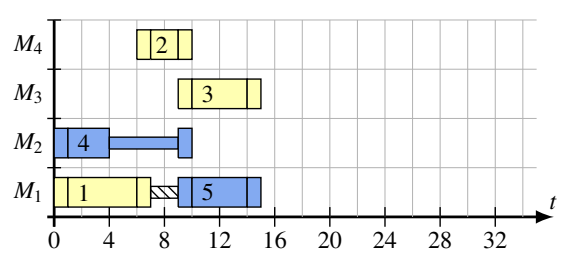

2) total flow time: 30

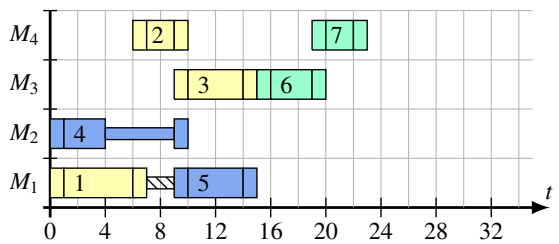

4) total flow time: 53

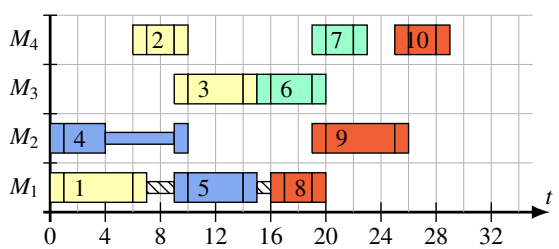

6) total flow time: 82

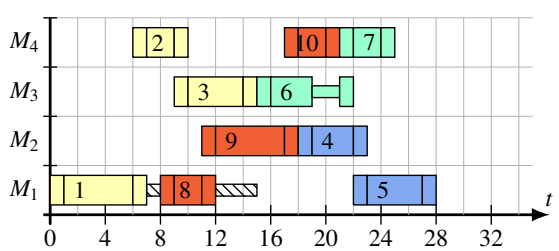

8) total flow time: 89

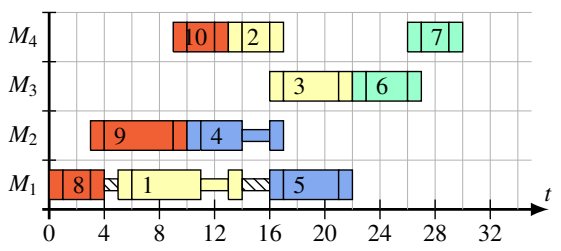

10) total flow time: 87

Fig. 8: Construction of an initial selection. 
objectives. We observed that JILS is quite robust with respect to different parameter settings.

Five independent runs of JILS were executed for each problem type, objective, and instance. The computation time limit of each run was set to 1800 seconds. In order to evaluate the approach also with shorter run times, we recorded the objective value of the best solution found so far by JILS after 60 and 300 seconds. The appendix contains the detailed results: for the JS in Table 5, 6, and 7, for the JSS in Table 8 and 9, and for the BJS in Table 10 and 11.

While we tried to keep the JILS as generic as possible, we incorporated a slight modification of the neighborhood for the JS with the makespan objective. We observed in preliminary tests that better results can be obtained for this problem type if JILS considers only the critical arcs at the border of so-called critical blocks. Indeed, it is well-known that swapping a critical arc in the "inner part" of a critical block does not improve the makespan. Therefore, we used this smaller neighborhood, called N5 in (Blazewicz et al, 1996), for the JS with the makespan objective.

\subsection{Comparison with benchmarks}

In order to assess the performance of JILS, we compare the attained results with current benchmarks from the literature when available. As these benchmarks were obtained using different computational settings, especially computing power and run times, we compare the average values of JILS (over the five runs) after 60, 300, and 1800 seconds with the benchmark values. While comparisons of heuristics are intrinsically difficult (see, e.g., Hooker, 1995), this test setting should provide evidence that the quality of the JILS results is comparable to the state-of-the-art for a large class of CJS-R problems. A simple performance measure is used to compare the results of JILS with benchmarks. For each benchmark result, we compute the relative gap of the average result of JILS over the five runs $(J I L S)$ with the result of the benchmark (bench), i.e., (JILS - bench)/bench. These gaps are determined for the results of JILS after a run time of 60,300 , and 1800 seconds. While this performance measure is simple to interpret and is widely used, some care should be given to the numbers as large gaps may be obtained if the benchmark value is low even if the absolute difference is small. We use small multiples (see, e.g., Tufte, 2001) to illustrate the relative gaps in a compact way.

We successively consider the JS, JSS, and BJS. In all three problem types, we first deal with the makespan objective and then address all other objectives.

\subsubsection{JS with the makespan objective}

There is a large collection of benchmark results available for the JS with the makespan objective. We briefly describe the approaches and the basic computational settings of the benchmarks we compare to.

As mentioned in Sect. 1, Grimes and Hebrard (2015) recently proposed a method that is applicable to a broad class of job shop scheduling problems with the makespan objective. We consider their average results (over 10 runs) for the JS obtained by their 
general light-weighted approach and abbreviate these results by $G H$. The authors used a Intel Pentium IV 3.0 GHz processor and a time limit of 3600 seconds per run.

Peng et al (2015) developed a specialized heuristic for the JS with the makespan objective. They combined a path relinking strategy with a tabu search, which uses a neighborhood based on swapping critical operations. Their approach is currently among the best heuristics for solving the JS with the makespan objective. We consider their average results over 10 runs (see Peng et al, 2015, Table 8, p. 160) and abbreviate these results by $P L C$. The authors used an AMD Athlon $3.0 \mathrm{GHz}$ processor, and the run time varied between 1 and 1726 seconds.

We first discuss the results obtained for the instances la01 to la40 and orb01 to orb10. The optimal values are known for these instances (see Brucker et al, 1994; Perregaard and Clausen, 1998; Grimes and Hebrard, 2015). Similar as other state-ofthe-art heuristics, JILS finds optimal or near-optimal solutions within a short computation time. Indeed, the overall average relative gap to the optimal values is $0.14 \%$, $0.06 \%$, and $0.03 \%$ for the results of JILS obtained after 60,300 , and 1800 seconds, respectively. Also, JILS finds an optimal solution in all the five runs for 41 instances (out of 50), and in at least one of the five runs for 47 instances.

The results of the Taillard instances are now compared with GH. The relative gaps of JILS to GH are illustrated in Fig. 9 (above) using small multiples as follows. The first, second, and third line depicts the relative gaps for the average results of JILS after 60, 300, and 1800 seconds, respectively. The time limit of JILS and the relative gap averaged over all instances is provided on the left. The range of the attained gaps is partitioned into seven intervals: $[a \% ;-6.0 \%],[-6.0 \% ;-2.0 \%],[-2.0 \% ;-0.5 \%]$, $[-0.5 \% ; 0.5 \%],[0.5 \% ; 2.0 \%],[2.0 \% ; 6.0 \%]$, and $[6.0 \% ; b \%]$, where $a$ is the minimum of $-10.0 \%$ and the minimum attained gap, and similarly, $b$ is the maximum of $10.0 \%$ and the maximum attained gap. Each instance is illustrated by drawing a small rectangle above the interval where its corresponding relative gap belongs to. In order to distinguish the instances, the rectangles are always drawn at the same relative position in each interval. For this purpose, an ordering of the instances is specified. To keep it simple, we use the orderings given in the result tables (see Table 5 to 11).

In Fig. 9 (above), we represent the first instance (ta01) at the top left (first row, first column) in each interval, then ta02 on its right (first row, second column), and so forth. Consider, for example, the instance ta42. As 10 instances are depicted per line, its position is in the fifth row and second column. The benchmark value of GH is 2027.5, and the average value obtained by JILS is 2033, 1990, and 1982 for 60, 300 , and 1800 seconds, respectively (see Table 5). This gives respective relative gaps of $0.3 \%,-1.8 \%$, and $-2.2 \%$. Hence, in the first, second, and third line of Fig. 9, the rectangle of ta 42 is drawn in the interval $[-0.5 \% ; 0.5 \%],[-2.0 \% ;-0.5 \%]$, and $[-6.0 \% ;-2.0 \%]$, respectively.

Considering the resulting small multiples in Fig. 9 (above), it can be observed that JILS is competitive with GH. Indeed, already after 60 seconds, the quality of JILS is comparable to GH. Furthermore, after 1800 seconds, JILS gives considerably lower results than $\mathrm{GH}$ for the largest instances (ta31 to ta50).

The following can be observed when comparing the relative gaps of JILS to PLC in Fig. 9 (below). JILS needs some time to get close to the values of PLC. After 60 seconds, PLC provides better results, especially for the large instances. Quite similar 

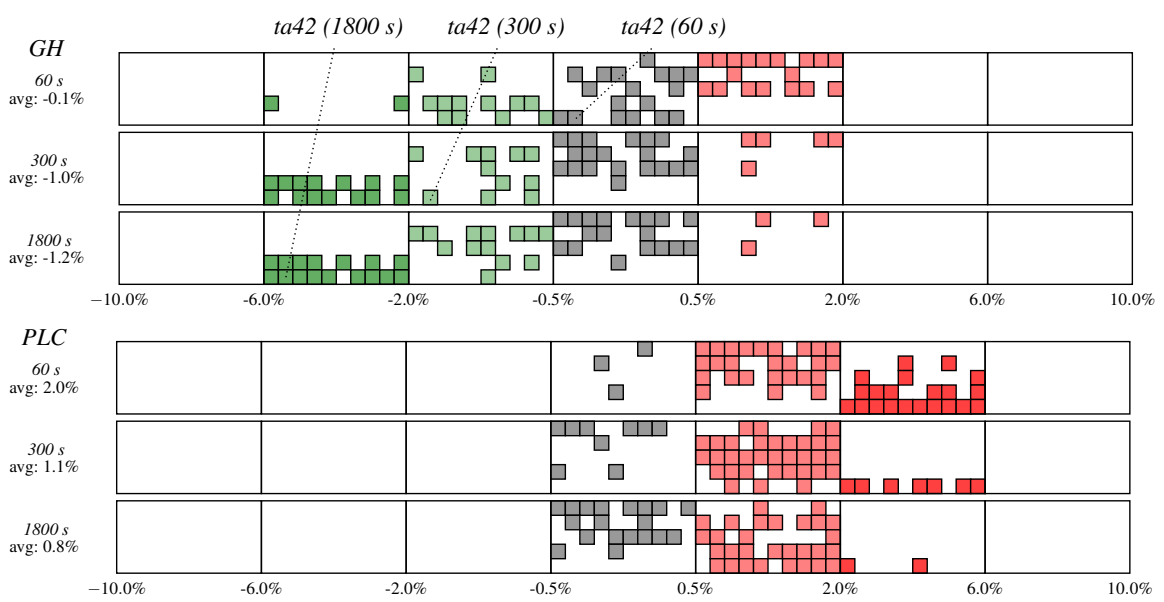

Fig. 9: Comparison of the results obtained in the JS with the makespan objective with results of Grimes and Hebrard (2015) (above) and Peng et al (2015) (below) in the Taillard instances.

results are attained after 1800 seconds. Indeed, the overall average gap is then $0.8 \%$. Only in some of the largest instances, PLC produces results that are more than $2 \%$ lower than the results of JILS. It can be concluded that JILS is quite competitive with PLC.

Table 1 provides a more detailed comparison of the JILS average results after 1800 seconds to the average results of GH and PLC and to the average results of Gonçalves and Resende (2014) (abbreviated by GR) and Zhang et al (2008) (abbreviated by ZRLG). GR and ZRLG also describe state-of-the-art approaches for the JS with the makespan objective. It can be verified that the specialized algorithms of PLC, GR, and ZRLG provide slightly better results than JILS, and JILS gives lower values than generic approach of $\mathrm{GH}$, especially for larger instances. For a more detailed comparison of the state-of-the-art approaches for the JS with the makespan objective, we refer to Peng et al (2015). Altogether, we conclude that JILS performs well in the JS with the makespan objective.

\subsubsection{JS with the other objectives}

The majority of the JS articles considers the makespan objective. Consequently, there are less benchmark results available for the other objectives. In this section, we successively consider the total flow time, total weighted tardiness, total squared flow time, and total tardiness objectives.

González et al (2010) developed a hybrid solution method for the JS with flow time objective. They combined a tabu search and a genetic algorithm. The tabu search applies a neighborhood in which a critical operation is moved (forward or backward) in its critical block provided that a sufficient condition for feasibility of the move is satisfied. We consider their average results over 20 runs (see González et al, 2010, 
Table 1: Comparison of the JILS average results after 1800 seconds (JILS) to current state-of-the-art results. Column one gives the instance name and columns two to six the results of JILS, GH, PLC, GR, and ZRLG. The values in brackets provide the relative gap (in \%) of the JILS result (res) to the benchmark value $(v a l)$, i.e., $(r e s-v a l) / v a l$. The last line presents the overall average relative gaps. All values are rounded to one decimal place.

\begin{tabular}{|c|c|c|c|c|c|}
\hline Instance & JILS & GH & PLC & GR & ZRLG \\
\hline ta01 & 1232.2 & $1231.0(0.1 \%)$ & $1231.0(0.1 \%)$ & $1231.0(0.1 \%)$ & $1231.0(0.1 \%)$ \\
\hline $\mathrm{ta} 02$ & 1245.4 & $1244.0(0.1 \%)$ & $1244.0(0.1 \%)$ & $1244.0(0.1 \%)$ & $1244.1(0.1 \%)$ \\
\hline ta03 & 1222.6 & $1218.0(0.4 \%)$ & $1218.0(0.4 \%)$ & $1218.0(0.4 \%)$ & $1219.4(0.3 \%)$ \\
\hline ta04 & 1177.4 & $1175.0(0.2 \%)$ & $1175.0(0.2 \%)$ & $1175.0(0.2 \%)$ & $1176.2(0.1 \%)$ \\
\hline ta05 & 1232.0 & $1224.0(0.7 \%)$ & $1224.0(0.7 \%)$ & $1224.9(0.6 \%)$ & $1224.0(0.7 \%)$ \\
\hline ta06 & 1242.2 & $1238.1(0.3 \%)$ & $1238.4(0.3 \%)$ & $1238.9(0.3 \%)$ & $1240.8(0.1 \%)$ \\
\hline ta07 & 1228.0 & $1227.0(0.1 \%)$ & $1228.0(0.0 \%)$ & $1228.0(0.0 \%)$ & $1228.0(0.0 \%)$ \\
\hline ta08 & 1217.8 & $1217.0(0.1 \%)$ & $1217.0(0.1 \%)$ & $1217.0(0.1 \%)$ & $1217.1(0.1 \%)$ \\
\hline ta09 & 1283.2 & $1274.0(0.7 \%)$ & $1274.0(0.7 \%)$ & $1277.0(0.5 \%)$ & $1275.2(0.6 \%)$ \\
\hline ta10 & 1245.0 & $1241.0(0.3 \%)$ & $1241.0(0.3 \%)$ & $1241.0(0.3 \%)$ & $1246.6(-0.1 \%)$ \\
\hline ta11 & 1374.6 & $1395.4(-1.5 \%)$ & $1359.9(1.1 \%)$ & $1360.0(1.1 \%)$ & $1367.6(0.5 \%)$ \\
\hline ta12 & 1375.4 & $1382.5(-0.5 \%)$ & $1369.9(0.4 \%)$ & $1372.6(0.2 \%)$ & $1374.3(0.1 \%)$ \\
\hline ta13 & 1355.2 & $1350.5(0.3 \%)$ & $1346.0(0.7 \%)$ & $1347.3(0.6 \%)$ & $1355.2(0.0 \%)$ \\
\hline ta14 & 1345.0 & $1345.1(0.0 \%)$ & $1345.0(0.0 \%)$ & $1345.0(0.0 \%)$ & $1346.7(-0.1 \%)$ \\
\hline ta15 & 1353.0 & $1371.4(-1.3 \%)$ & $1339.0(1.0 \%)$ & $1348.9(0.3 \%)$ & $1348.4(0.3 \%)$ \\
\hline ta16 & 1368.6 & $1392.1(-1.7 \%)$ & $1360.0(0.6 \%)$ & $1362.1(0.5 \%)$ & $1366.2(0.2 \%)$ \\
\hline ta17 & 1476.0 & $1477.0(-0.1 \%)$ & $1473.0(0.2 \%)$ & $1470.5(0.4 \%)$ & $1472.9(0.2 \%)$ \\
\hline ta18 & 1417.8 & $1435.6(-1.2 \%)$ & $1401.0(1.2 \%)$ & $1400.9(1.2 \%)$ & $1408.7(0.6 \%)$ \\
\hline ta19 & 1344.8 & $1359.6(-1.1 \%)$ & $1336.6(0.6 \%)$ & $1333.2(0.9 \%)$ & $1340.6(0.3 \%)$ \\
\hline ta 20 & 1363.0 & $1372.6(-0.7 \%)$ & $1351.3(0.9 \%)$ & $1350.4(0.9 \%)$ & $1356.7(0.5 \%)$ \\
\hline ta21 & 1660.2 & $1656.3(0.2 \%)$ & $1645.2(0.9 \%)$ & $1647.0(0.8 \%)$ & $1650.5(0.6 \%)$ \\
\hline ta22 & 1618.8 & $1623.8(-0.3 \%)$ & $1603.8(0.9 \%)$ & $1600.0(1.2 \%)$ & $1606.4(0.8 \%)$ \\
\hline ta23 & 1566.4 & $1574.6(-0.5 \%)$ & $1559.6(0.4 \%)$ & $1562.6(0.2 \%)$ & $1564.5(0.1 \%)$ \\
\hline ta24 & 1660.6 & $1648.6(0.7 \%)$ & $1647.7(0.8 \%)$ & $1650.6(0.6 \%)$ & $1653.2(0.4 \%)$ \\
\hline ta25 & 1604.2 & $1613.5(-0.6 \%)$ & $1597.0(0.5 \%)$ & $1602.0(0.1 \%)$ & $1607.7(-0.2 \%)$ \\
\hline ta26 & 1658.8 & $1671.5(-0.8 \%)$ & $1651.4(0.4 \%)$ & $1652.3(0.4 \%)$ & $1654.9(0.2 \%)$ \\
\hline ta27 & 1694.2 & $1695.8(-0.1 \%)$ & $1686.7(0.4 \%)$ & $1685.6(0.5 \%)$ & $1688.7(0.3 \%)$ \\
\hline ta28 & 1623.8 & $1619.0(0.3 \%)$ & $1616.2(0.5 \%)$ & $1611.7(0.8 \%)$ & $1616.6(0.4 \%)$ \\
\hline ta29 & 1632.6 & $1638.8(-0.4 \%)$ & $1627.4(0.3 \%)$ & $1627.4(0.3 \%)$ & $1630.1(0.2 \%)$ \\
\hline ta30 & 1602.6 & $1607.4(-0.3 \%)$ & $1588.3(0.9 \%)$ & $1588.5(0.9 \%)$ & $1597.7(0.3 \%)$ \\
\hline ta31 & 1765.6 & $1826.5(-3.3 \%)$ & $1764.0(0.1 \%)$ & $1764.4(0.1 \%)$ & $1765.8(0.0 \%)$ \\
\hline ta32 & 1828.8 & $1884.8(-3.0 \%)$ & $1803.5(1.4 \%)$ & $1794.1(1.9 \%)$ & $1811.7(0.9 \%)$ \\
\hline ta33 & 1817.8 & $1886.9(-3.7 \%)$ & $1794.6(1.3 \%)$ & $1793.7(1.3 \%)$ & $1806.7(0.6 \%)$ \\
\hline ta 34 & 1842.6 & $1910.6(-3.6 \%)$ & $1831.2(0.6 \%)$ & $1832.1(0.6 \%)$ & $1831.8(0.6 \%)$ \\
\hline ta35 & 2007.0 & $2007.0(0.0 \%)$ & $2007.0(0.0 \%)$ & $2007.0(0.0 \%)$ & $2011.0(-0.2 \%)$ \\
\hline ta36 & 1828.2 & $1886.6(-3.1 \%)$ & $1819.0(0.5 \%)$ & $1822.9(0.3 \%)$ & $1820.5(0.4 \%)$ \\
\hline ta37 & 1799.6 & $1831.4(-1.7 \%)$ & $1776.8(1.3 \%)$ & $1777.8(1.2 \%)$ & $1784.4(0.9 \%)$ \\
\hline ta38 & 1687.8 & $1739.0(-2.9 \%)$ & $1673.0(0.9 \%)$ & $1676.7(0.7 \%)$ & $1678.5(0.6 \%)$ \\
\hline ta39 & 1805.2 & $1832.4(-1.5 \%)$ & $1795.0(0.6 \%)$ & $1801.6(0.2 \%)$ & $1806.6(-0.1 \%)$ \\
\hline ta 40 & 1706.2 & $1777.2(-4.0 \%)$ & $1676.0(1.8 \%)$ & $1678.1(1.7 \%)$ & $1684.0(1.3 \%)$ \\
\hline ta41 & 2059.8 & $2117.2(-2.7 \%)$ & $2018.6(2.0 \%)$ & $2018.7(2.0 \%)$ & $2028.5(1.5 \%)$ \\
\hline ta 42 & 1981.6 & $2027.5(-2.3 \%)$ & $1950.3(1.6 \%)$ & $1949.3(1.7 \%)$ & $1964.5(0.9 \%)$ \\
\hline ta43 & 1896.8 & $1958.0(-3.1 \%)$ & $1865.1(1.7 \%)$ & $1863.1(1.8 \%)$ & $1882.6(0.8 \%)$ \\
\hline ta44 & 2016.6 & $2100.8(-4.0 \%)$ & $1989.1(1.4 \%)$ & $1992.4(1.2 \%)$ & $1998.2(0.9 \%)$ \\
\hline ta45 & 2020.4 & $2077.9(-2.8 \%)$ & $2000.5(1.0 \%)$ & $2000.0(1.0 \%)$ & $2006.8(0.7 \%)$ \\
\hline ta 46 & 2069.0 & $2106.4(-1.8 \%)$ & $2022.3(2.3 \%)$ & $2015.5(2.7 \%)$ & $2029.2(2.0 \%)$ \\
\hline ta47 & 1942.4 & $1994.1(-2.6 \%)$ & $1906.2(1.9 \%)$ & $1902.1(2.1 \%)$ & $1918.2(1.3 \%)$ \\
\hline ta 48 & 1987.0 & $2055.4(-3.3 \%)$ & $1955.5(1.6 \%)$ & $1959.2(1.4 \%)$ & $1968.6(0.9 \%)$ \\
\hline ta 49 & 2010.6 & $2053.9(-2.1 \%)$ & $1971.5(2.0 \%)$ & $1972.6(1.9 \%)$ & $1980.0(1.5 \%)$ \\
\hline ta50 & 1965.6 & $2039.7(-3.6 \%)$ & $1931.4(1.8 \%)$ & $1927.0(2.0 \%)$ & $1945.6(1.0 \%)$ \\
\hline \multicolumn{2}{|c|}{ overall average } & $-1.3 \%$ & $0.8 \%$ & $0.8 \%$ & $0.4 \%$ \\
\hline
\end{tabular}


GVSV

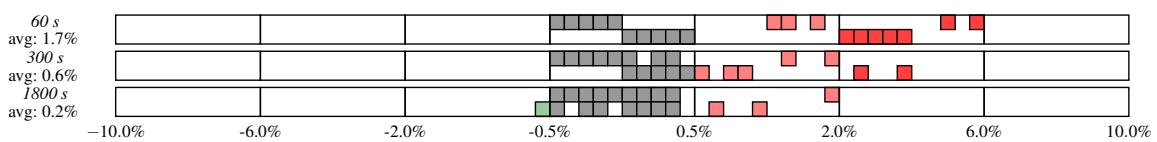

Fig. 10: Comparison of the results obtained in the JS with total flow time objective with results of González et al (2010).

Table 4, p. 41) and abbreviate these by GVSV. The authors used an Intel Xeon 2.66 $\mathrm{GHz}$ processor, and the computation time per run varied between 98 and 895 seconds.

Fig. 10 illustrates the relative gaps of JILS to GVSV for the instances la01 to la20. It can be observed that JILS gives slightly higher values than GVSV after 60 seconds. After 1800 seconds, the performances of JILS and GVSV are similar. Indeed, the average relative gap of JILS to GVSV is then only $0.2 \%$.

A considerable amount of work has been dedicated to study the JS with total weighted tardiness objective, which is a generalization of the JS with total flow time objective. We compare JILS with the following two approaches.

As mentioned in Sect. 1, Mati et al (2011) recently developed a tabu search method that is applicable to the JS with a general regular objective. We consider their results for the JS with total weighted tardiness objective (see Mati et al, 2011, results with $f=1.3$ in Table 3, p. 38) and abbreviate these results by $M D L$. The authors used a $2.6 \mathrm{GHz}$ processor. As they set the computation time limit to only 18 seconds per run, we compare the JILS results to their best results over 10 runs.

González et al (2012a) recently addressed the JSS problem with weighted tardiness objective, and they also provide benchmark results for the JS (without setup times). They proposed a hybrid heuristic, combining a tabu search and a genetic algorithm. In the tabu search component, neighbors are built by reversing a single critical arc provided that some feasibility condition is satisfied. We consider their average results over 10 runs (see González et al, 2012a, column GTN with $f=1.3$, Table 2, p. 2108 and Table 3, p. 2110) and abbreviate these results by $G G V V$. They used an Intel Core 2 Duo processor with $2.66 \mathrm{GHz}$, and the computation time of a run varied between 18 and 1931 seconds.

Fig. 11 (upper part) illustrates the relative gaps of JILS to MDL for the instances la17 to la20 and orb01 to orb10. It can be observed that, after 300 and 1800 seconds, the results of JILS are quite similar as MDL. Hence, we conclude that both methods have a similar performance.

Fig. 11 (lower part) illustrates the relative gaps of JILS to GGVV for the instances la01 to la40 and orb01 to orb10. It can be observed that JILS quickly finds results of a similar quality than GGVV for the small instances. Furthermore, after 1800 seconds, the average relative gap is $0.0 \%$. Interestingly, the relative gaps are quite high for some instances. They are, for example, $-7.5 \%$ for instance la34 and $7.3 \%$ for la29 after 1800 seconds.

For the total squared flow time and total tardiness objectives, no benchmarks results are available. Hence, we resorted to compare the performance of JILS with results obtained via a mixed integer programming (MIP) model that we derived in a 
$M D L$
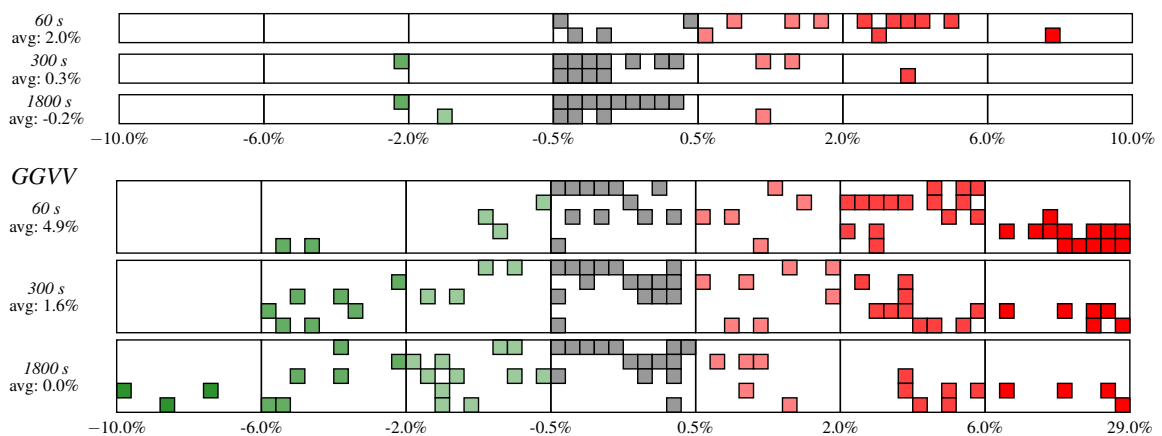

Fig. 11: Comparison of the results obtained in the JS with total weighted tardiness objective with results of Mati et al (2011) (above) and González et al (2012a) (below).

Table 2: Comparison of the MIP results to the JILS results for JS instances with total squared flow time objective (left, in units of 1000) and total tardiness objective (right). Columns avg-1800 provide the results obtained by JILS after 1800 seconds, columns LB; UB give the MIP lower and upper bounds ("opt" if they are the same), and columns diff present the relative difference of the results avg-1800 and UB. The instances are grouped according to their size $n \times m$, where $n$ is the number of jobs and $m$ the number of machines.

\begin{tabular}{|c|c|c|c|c|c|c|}
\hline \multirow{2}{*}{$\begin{array}{l}\text { objective } \\
\text { results }\end{array}$} & \multicolumn{3}{|c|}{ total squared flow time } & \multicolumn{3}{|c|}{ total tardiness } \\
\hline & avg-1800 & LB ; UB & diff & avg-1800 & $\mathrm{LB} ; \mathrm{UB}$ & diff \\
\hline \multicolumn{7}{|l|}{$10 \times 5$} \\
\hline la01 & 2599 & 2599 (opt) & $0.0 \%$ & 1194 & 1194 (opt) & $0.0 \%$ \\
\hline $\mathrm{la} 02$ & 2296 & $2110 ; 2339$ & $-1.8 \%$ & 1065 & 1065 (opt) & $0.0 \%$ \\
\hline la03 & 1956 & 1956 (opt) & $0.0 \%$ & 1076 & 1076 (opt) & $0.0 \%$ \\
\hline $\mathrm{la} 04$ & 2060 & $1916 ; 2060$ & $0.0 \%$ & 1096 & 1096 (opt) & $0.0 \%$ \\
\hline la05 & 1862 & 1862 (opt) & $0.0 \%$ & 1164 & 1164 (opt) & $0.0 \%$ \\
\hline \multicolumn{7}{|l|}{$15 \times 5$} \\
\hline la06 & 5953 & $2193 ; 6396$ & $-6.9 \%$ & 3491 & $916 ; 3643$ & $-4.2 \%$ \\
\hline la07 & 5252 & $2022 ; 5651$ & $-7.1 \%$ & 3282 & $713 ; 3333$ & $-1.5 \%$ \\
\hline la08 & 5188 & $1969 ; 6135$ & $-15.4 \%$ & 3056 & $735 ; 3872$ & $-21.1 \%$ \\
\hline la09 & 6553 & $2364 ; 7340$ & $-10.7 \%$ & 3567 & $786 ; 3984$ & $-10.5 \%$ \\
\hline la10 & 6348 & $2150 ; 6916$ & $-8.2 \%$ & 3655 & $818 ; 4097$ & $-10.8 \%$ \\
\hline
\end{tabular}

straightforward manner from the disjunctive programming formulation of the CJS-R problem (see Sect. 2.1). As preliminary tests revealed that the simple MIP approach only finds good solutions in small instances, we decided to use the smallest instances la01 to la10 for these experiments. We ran the MIP using the solver Gurobi 6.5 with a time limit of 7200 seconds for each instance. Table 2 reports the obtained results. The following can be observed for the instances of size $10 \times 5$. The MIP approach shows that JILS always found an optimal solution for the total tardiness objective. For the total squared flow time objective, the MIP approach could prove optimality of three (out of five) results, and JILS obtained results with a similar quality. For the instances of size $15 \times 5$, the optimality gaps of the MIP approach are large. On average, JILS 
gives $9.7 \%$ and $9.6 \%$ lower results than the MIP approach for the total squared flow time and the total tardiness objective, respectively.

In summary, JILS is competitive in the JS with all considered objectives.

\subsubsection{JSS with the makespan objective}

The JSS has attracted the attention of many researchers. As in the JS, most articles discuss the makespan objective.

A prominent contribution addressing the JSS with the makespan objective is (Balas et al, 2008), in which a shifting bottleneck procedure is proposed. The single machine scheduling subproblems are treated as traveling salesman problems with time windows, which are solved with dynamic programming. We consider the results obtained by the multi-run SB-RGLS10 version (see Balas et al, 2008, Table 6, p. 2108 and Table 3, p. 260) and abbreviate these results by BSV. The authors used a UltraSPARC-II processor with $360 \mathrm{MHz}$, and the computation time of a run varied between 264 and 3033 seconds.

Vela et al (2010) also addressed the JSS with the makespan objective. They proposed a hybrid heuristic, which combines a local search and a genetic algorithm. In the tabu search, neighbors are built by reversing the orders of operations in a critical block. They consider only moves where feasibility is ensured by some sufficient condition. We consider their average results over 30 runs (see Vela et al, 2010, Table 5, p. 161 and Table 6, p. 162) and abbreviate these results by VVG. They used an Intel Pentium IV processor with $1.7 \mathrm{GHz}$, and the computation time of a run varied between 2 and 166 seconds.

González et al (2012b) tackled the JSS with the makespan objective. They proposed a genetic algorithm with a local search component. We consider their average results (over 30 runs) obtained by Lamarckian evolution (see González et al, 2012b, Table 1, p. 157) and abbreviate these results by GVV. They used an Intel Core 2 Duo processor with $2.6 \mathrm{GHz}$, and the computation time of a run varied between 35 and 180 seconds.

Fig. 12 (upper part) illustrates the relative gaps of JILS to BSV for the instances t2-ps01 to t2-ps15. We observe that the average gaps are small and conclude that the results of JILS are of a similar quality than BSV.

Fig. 12 (middle part) illustrates the relative gaps of JILS to VVG for the instances t2-ps01 to t2-ps15 and t2-laXYsdst, $X Y \in\{21,24,25,27,29,38,40\}$. It can be observed that the results of JILS are slightly better than VVG, even after a short run time.

Fig. 12 (lower part) illustrates the relative gaps of JILS to GVV for the instances t2-ps11 to t2-ps15, t2-laXY sdst, $X Y \in\{21,24,25,27,29,38,40\}$, t2-abzZsdst, $Z \in$ $\{7,8,9\}$. It can be seen that GVV provides better results than JILS, especially when compared to the JILS results obtained after 60 and 300 seconds. Nevertheless, the average gap is only about $1.8 \%$ after 1800 seconds.

In summary, JILS has a quite good performance in the JSS with the makespan objective. 
BSV

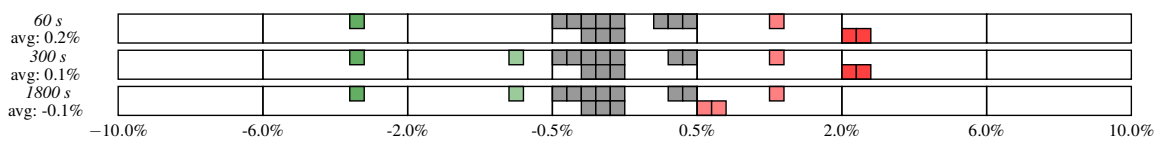

$V V G$

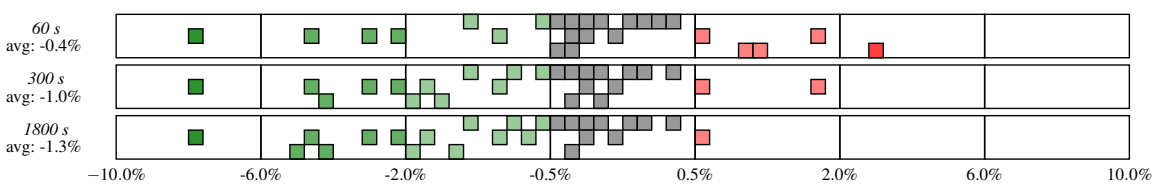

GVV

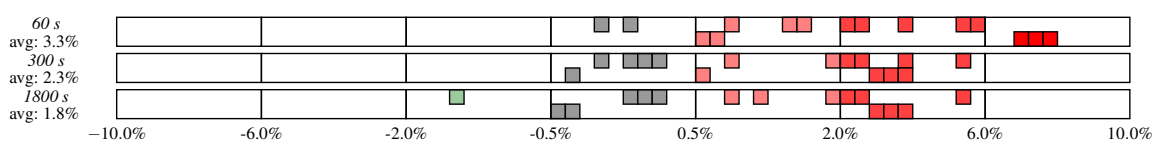

Fig. 12: Comparison of the results obtained in the JSS with the makespan objective to the results of Balas et al (2008); Vela et al (2010); González et al (2012b).

GGVV2

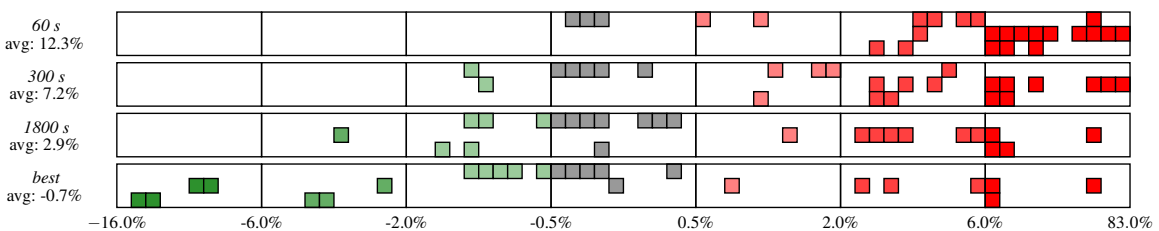

Fig. 13: Comparison of the results obtained in the JSS with total weighted tardiness objective to the results of González et al (2012a).

\subsubsection{JSS with the other objectives}

Only few papers address the JSS with other objectives than the makespan.

González et al (2012a) tackle the JSS with total weighted tardiness objective. The approach was already described in Sect. 5.4.2. We consider their average results over 10 runs (González et al, 2012a, column GTN with $f=1.6$ in Table 1, p. 2106) and abbreviate these results by GGVV2. They used an Intel Core 2 Duo processor with $2.66 \mathrm{GHz}$, and the computation time of a run varied between 45 and 810 seconds.

Fig. 13 illustrates the relative gaps of JILS to GGVV2 for the instances t2-ps01 to t2-ps15, t2-la $X Y$ sdst, $X Y \in\{21,24,25,27,29,38,40\}$, and t2-abzZsdst, $Z \in\{7,8,9\}$. It can be seen that GGVV2 provides substantially lower values when compared to the average results of JILS after 60 and 300 seconds. Also, when compared to the results obtained after 1800 seconds, GGVV2 gives $2.9 \%$ lower results on average. However, as illustrated in the last row, the best results of JILS (over the five runs) meet the performance of GGVV2. We recognized in preliminary tests that JILS can be made 
Table 3: Comparison of the MIP results to the results of JILS for JSS instances with total flow time (top left), total squared flow time (top right, in units of 1000), and total tardiness objective (bottom). See Table 2 for a detailed explanation.

\begin{tabular}{|c|c|c|c|c|c|c|}
\hline \multirow{2}{*}{$\begin{array}{l}\text { objective } \\
\text { results }\end{array}$} & \multicolumn{3}{|c|}{ total flow time } & \multicolumn{3}{|c|}{ total squared flow time } \\
\hline & avg-1800 & LB ; UB & diff & avg-1800 & $\mathrm{LB} ; \mathrm{UB}$ & diff \\
\hline \multicolumn{7}{|l|}{$10 \times 5$} \\
\hline t2-ps01 & 6050 & 6050 (opt) & $0.0 \%$ & 4053 & $3753 ; 4210$ & $-3.7 \%$ \\
\hline $\mathrm{t} 2-\mathrm{ps} 02$ & 5505 & $5220 ; 5522$ & $-0.3 \%$ & 3479 & $2511 ; 3479$ & $0.0 \%$ \\
\hline $\mathrm{t} 2-\mathrm{ps} 03$ & 5092 & $4773 ; 5171$ & $-1.5 \%$ & 2973 & $2467 ; 2973$ & $0.0 \%$ \\
\hline t2-ps04 & 5478 & $4793 ; 5549$ & $-1.3 \%$ & 3375 & $2449 ; 3467$ & $-2.7 \%$ \\
\hline $\mathrm{t} 2-\mathrm{ps} 05$ & 5041 & 5041 (opt) & $0.0 \%$ & 2874 & $2173 ; 3054$ & $-5.9 \%$ \\
\hline \multicolumn{7}{|l|}{$15 \times 5$} \\
\hline $\mathrm{t} 2-\mathrm{ps} 06$ & 10706 & $6522 ; 11261$ & $-4.9 \%$ & 8685 & $2756 ; 10690$ & $-18.8 \%$ \\
\hline t2-ps07 & 10144 & $5936 ; 11211$ & $-9.5 \%$ & 7912 & $2458 ; 9349$ & $-15.4 \%$ \\
\hline t2-ps08 & 10193 & $6231 ; 11074$ & $-8.0 \%$ & 8019 & $2620 ; 9937$ & $-19.3 \%$ \\
\hline t2-ps09 & 10879 & $6578 ; 12253$ & $-11.2 \%$ & 9346 & $2849 ; 10564$ & $-11.5 \%$ \\
\hline $\mathrm{t} 2-\mathrm{ps} 10$ & 10828 & $6324 ; 11709$ & $-7.5 \%$ & 8950 & $2731 ; 10433$ & $-14.2 \%$ \\
\hline
\end{tabular}

\begin{tabular}{llll}
\hline \multirow{2}{*}{$\begin{array}{l}\text { objective } \\
\text { results }\end{array}$} & \multicolumn{3}{c}{ total tardiness } \\
\cline { 2 - 4 } & avg-1800 & LB $;$ UB & diff \\
\hline $10 \times 5$ & & & \\
t2-ps01 & 1623 & $1623(\mathrm{opt})$ & $0.0 \%$ \\
t2-ps02 & 1395 & $1395(\mathrm{opt})$ & $0.0 \%$ \\
t2-ps03 & 1361 & $1361(\mathrm{opt})$ & $0.0 \%$ \\
t2-ps04 & 1583 & $1020 ; 1620$ & $-2.3 \%$ \\
t2-ps05 & 1545 & $1190 ; 1553$ & $-0.5 \%$ \\
$15 \times 5$ & & & \\
t2-ps06 & 4379 & $591 ; 5241$ & $-16.4 \%$ \\
t2-ps07 & 4180 & $747 ; 4521$ & $-7.5 \%$ \\
t2-ps08 & 4083 & $537 ; 4966$ & $-17.8 \%$ \\
t2-ps09 & 4155 & $976 ; 4672$ & $-11.1 \%$ \\
t2-ps10 & 4305 & $807 ; 4897$ & $-12.1 \%$ \\
\hline
\end{tabular}

more robust for the JSS with total weighted tardiness if a specialized neighborhood is used. A further investigation is left for future work.

No benchmarks results are available for the total flow time, total squared flow time, and total tardiness objectives. Hence, as for the JS, we compare the performance of JILS with results obtained by a MIP approach (see also Sect. 5.4.2). For this purpose, we considered the smallest JSS instances ts-ps01 to ts-ps10, and ran the MIP with the solver Gurobi 6.5 using a time limit of 7200 seconds per instance. Table 3 reports the obtained results. The following can be observed for the instances of size $10 \times 5$. The average results of JILS are equal as or lower than the MIP results. Also, for the instances where the MIP approach could prove optimality, JILS always found an optimal solution. For the instances of size $15 \times 5$, JILS gives substantially lower results than the MIP approach. Indeed, the average relative gap is $-8.2 \%,-15.8 \%$, and $-13.0 \%$ for the total flow time, total squared flow time, and total tardiness objective, respectively.

Altogether, we conclude that JILS performs well in the JSS with all considered objectives. 


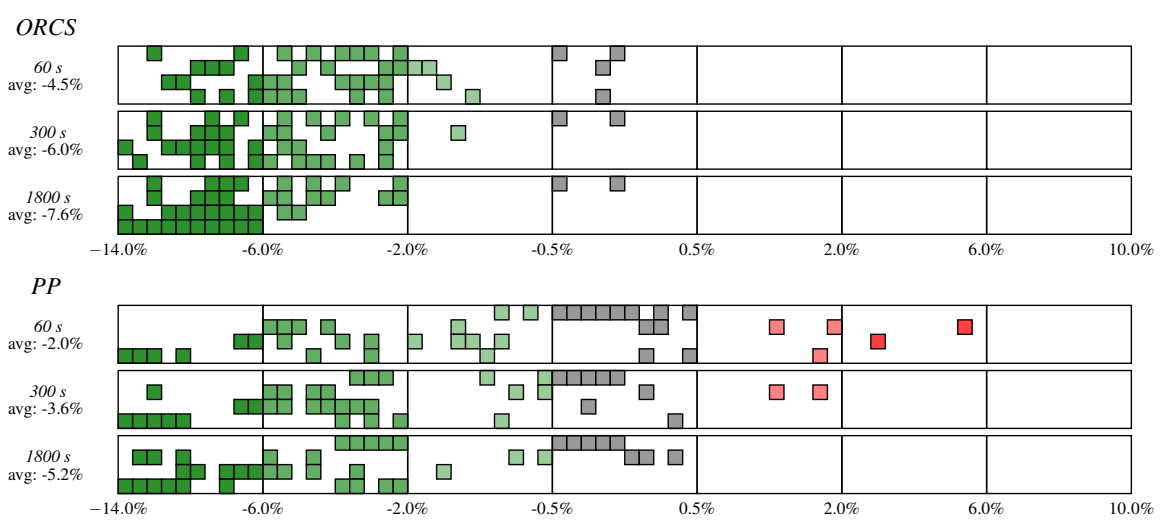

Fig. 14: Comparison of the results obtained in the BJS with the makespan objective to results of Oddi et al (2012); Pranzo and Pacciarelli (2015).

\subsubsection{BJS with the makespan objective}

A considerable amount of work has been dedicated to the BJS with the makespan objective. The benchmarks are taken from the following two recently published articles.

Oddi et al (2012) developed a constraint programming approach for the BJS with the makespan objective. They proposed an improvement algorithm based on iterative flattening search, and they performed tests with the IBM ILOG CP optimizer. We consider their results obtained by the CP optimizer (see Oddi et al, 2012, Table 3, p. 7) and abbreviate these results by ORCS. They used an AMD Phenom II X4 Quad processor with $3.5 \mathrm{GHz}$, and executed one run of 1800 seconds for each instance.

Pranzo and Pacciarelli (2015) developed an iterated greedy algorithm for the BJS with the makespan objective. Their approach is based on a repetition of a destruction phase, which is deleting a part of the solution, and a construction phase, which starts from a partial solution and applies a greedy approach to construct a new solution. We consider their results from (Pranzo and Pacciarelli, 2015, Table 7, p. 21) and abbreviate them by PP. They used an Intel Core 2 Duo processor with $2.6 \mathrm{GHz}$. As they set the computation time limit to only 60 seconds per run, we compare the average results of JILS with their best results over 10 runs.

Fig. 14 illustrates the relative gaps of JILS to ORCS (above) and PP (below) for the instances la01 to la40. It can be observed that JILS is substantially better than ORCS. Indeed, the average gap is $-4.5 \%,-6.0 \%$, and $-7.6 \%$ after 60,300 , and 1800 seconds, respectively. Especially in the large instances, JILS gives substantially better results than ORCS. The average gap is, for example, $-12.0 \%$ for the instances of size $15 \times 15$ (i.e., la36 to la40).

Considering the results of PP, it can be seen that JILS gives substantially lower values. Indeed, the average gap is $-2.0 \%,-3.6 \%$, and $-5.2 \%$ after 60,300 , and 1800 seconds, respectively. JILS is at a particular advantage in large instances. The results of JILS are, on average, $13.3 \%$ lower than PP for the instances of size $30 \times 10$ (i.e., la31 to la35). 
We conclude that JILS is substantially improving the best results of the literature in the BJS with the makespan objective.

Note that we proposed a tabu search in (Gröflin et al, 2011) for the BJS with machine flexibility and the makespan objective, and a refined version for more general complex job shops with the makespan criterion in (Bürgy, 2014). These methods formed the basis for the development of JILS, and the attained results in (Bürgy, 2014) are of a similar quality than the present results.

\subsubsection{BJS with the other objectives}

To the best our knowledge, no benchmark results are available for all other objectives. Therefore, we compare the performance of JILS to the results obtained by the same MIP approach that was applied for the JS and JSS (see Sect. 5.4.2). For this purpose, we considered the smallest BJS instances la01 to la10, and ran the MIP with the solver Gurobi 6.5 using a time limit of 7200 seconds per instance. Table 4 reports the obtained results.

The following can be observed for the instances of size $10 \times 5$. The MIP approach shows that JILS always found an optimal solution for the total flow time, total tardiness, and total weighted tardiness objectives. In addition, the JILS results are of the same quality or slightly better than the MIP results for the total squared flow time objective. Similar as in the JS and JSS, the optimality gaps of the MIP results are large for the instances of size $15 \times 5$ for all four objectives, and JILS gives substantially lower results.

In summary, JILS has a good performance for the BJS with all considered objectives.

\section{Concluding remarks}

We developed a neighborhood that can be applied to a wide range of job shop scheduling problems with regular objectives. By casting the neighborhood in a tabu search, we evaluated its performance with an extensive experimental study using three wellknown job shop scheduling problems and five regular objectives. The obtained results show that the proposed approach has a good, robust performance. Indeed, it is quite competitive with the state-of-the-art for all considered objectives in the job shop and in the job shop with sequence-dependent setup times, it substantially improves the state-of-the-art results in the blocking job shop with the makespan objective, and it establishes first results in the blocking job shop with the other considered objectives.

The class of regular objectives is certainly valuable in practice. The total squared tardiness objective makes it possible, for example, to keep the maximum tardiness low while maintaining an acceptable level for the total tardiness, which cannot be accomplished by a linear (weighted) penalization of the tardiness. In further research, it could be interesting to investigate non-regular objectives, which, for example, penalize earliness and tardiness. This type of objectives is especially relevant in a justin-time scheduling context. 
Table 4: Comparison of the MIP results to JILS for the BJS instances with total flow time objective (top left), total squared flow time objective (top right, in units of 1000), total tardiness objective (bottom left), and total weighted tardiness objective (bottom right). See Table 2 for a detailed explanation.

\begin{tabular}{|c|c|c|c|c|c|c|}
\hline \multirow{2}{*}{$\begin{array}{l}\text { objective } \\
\text { results }\end{array}$} & \multicolumn{3}{|c|}{ total flow time } & \multicolumn{3}{|c|}{ total squared flow time } \\
\hline & avg-1800 & $\mathrm{LB} ; \mathrm{UB}$ & diff & avg-1800 & $0 \quad \mathrm{LB}$; UB & diff \\
\hline \multicolumn{7}{|l|}{$10 \times 5$} \\
\hline la01 & 5152 & 5152 (opt) & $0.0 \%$ & 3123 & $2928 ; 3123$ & $0.0 \%$ \\
\hline la02 & 4937 & 4937 (opt) & $0.0 \%$ & 2982 & $2313 ; 2982$ & $0.0 \%$ \\
\hline $\mathrm{la} 03$ & 4605 & 4605 (opt) & $0.0 \%$ & 2574 & $2108 ; 2682$ & $-4.0 \%$ \\
\hline la04 & 4764 & 4764 (opt) & $0.0 \%$ & 2663 & $2318 ; 2663$ & $0.0 \%$ \\
\hline la05 & 4447 & 4447 (opt) & $0.0 \%$ & 2309 & $1816 ; 2309$ & $0.0 \%$ \\
\hline \multicolumn{7}{|l|}{$15 \times 5$} \\
\hline la06 & 9715 & $5705 ; 10721$ & $-9.4 \%$ & 7769 & $2356 ; 10296$ & $-24.5 \%$ \\
\hline la07 & 9136 & $5301 ; 10310$ & $-11.4 \%$ & 6715 & $1899 ; 8944$ & $-24.9 \%$ \\
\hline la08 & 9042 & $5716 ; 11051$ & $-18.2 \%$ & 6722 & $2080 ; 9840$ & $-31.7 \%$ \\
\hline la09 & 10237 & $6001 ; 11169$ & $-8.3 \%$ & 8690 & $2276 ; 14300$ & $-39.2 \%$ \\
\hline la10 & 9758 & $5881 ; 10684$ & $-8.7 \%$ & 7986 & $2417 ; 10650$ & $-25.0 \%$ \\
\hline \multirow{2}{*}{$\begin{array}{l}\text { objective } \\
\text { results }\end{array}$} & \multicolumn{2}{|c|}{ total tardiness } & & \multicolumn{2}{|c|}{ total weighted tardiness } & \\
\hline & avg-1800 & LB ; UB & diff & avg-1800 l & $\mathrm{LB} ; \mathrm{UB}$ & diff \\
\hline \multicolumn{7}{|l|}{$10 \times 5$} \\
\hline la01 & 743 & 743 (opt) & $0.0 \%$ & 1486 & 1486 (opt) & $0.0 \%$ \\
\hline $\mathrm{la} 02$ & 700 & 700 (opt) & $0.0 \%$ & 1251 & 1251 (opt) & $0.0 \%$ \\
\hline la03 & 736 & 736 (opt) & $0.0 \%$ & 1461 & 1461 (opt) & $0.0 \%$ \\
\hline la04 & 716 & 716 (opt) & $0.0 \%$ & 1236 & 1236 (opt) & $0.0 \%$ \\
\hline la05 & 796 & 796 (opt) & $0.0 \%$ & 1244 & 1244 (opt) & $0.0 \%$ \\
\hline \multicolumn{7}{|l|}{$15 \times 5$} \\
\hline la06 & 3075 & $165 ; 3475$ & $-11.5 \%$ & 5469 & $680 ; 7069$ & $-22.6 \%$ \\
\hline la07 & 2946 & $402 ; 3764$ & $-21.7 \%$ & 4727 & $748 ; 6634$ & $-28.7 \%$ \\
\hline la08 & 2831 & $387 ; 3260$ & $-13.1 \%$ & 4951 & $872 ; 6049$ & $-18.1 \%$ \\
\hline la09 & 3127 & $212 ; 3762$ & $-16.9 \%$ & 5685 & $942 ; 7656$ & $-25.7 \%$ \\
\hline la10 & 2994 & $291 ; 3953$ & $-24.3 \%$ & 5785 & $561 ; 6275$ & $-7.8 \%$ \\
\hline
\end{tabular}

The proposed neighbor generation scheme provides a general tool to derive a large set of feasible neighbors. It may be interesting to consider other types of neighbors than those used in the proposed neighborhood. Introducing larger moves of operations within a critical block may be promising. It could also be interesting to consider the optimal (re-) insertion of a job instead of searching for a neighbor close to the current solution. This optimal job insertion problem may be used, for example, to construct a good initial solution and in a large neighborhood search approach. Although the optimal job insertion problem is already NP-hard for the classical job shop, the nice characterization of all feasible insertions given in Sect. 3 may help to develop an efficient method that inserts a job in a near-optimal fashion.

Acknowledgements The author gratefully acknowledges the constructive remarks of three anonymous referees which led to several improvements in the presentation. This research is partially funded by the Swiss National Science Foundation Grant P2FRP2 161720. 


\section{Appendix}

\subsection{Proofs of job insertion properties}

Hereafter, we provide proofs of Proposition 2, Theorem 1, and Theorem 2. Although they can easily be derived from Gröflin and Klinkert (2007), we give them for convenience and completeness

Proof (of Proposition 2) (i) Observe that any acyclic insertion $T$ is stable in $H$ by construction of $H$. If insertion $T$ is also complete hence feasible, it is of size $\left|E^{J}\right| / 2$ as $|T \cap\{e, \bar{e}\}|=1$ for each pair $\in \mathscr{E}^{J}$ by (9). Then $|T|=\left|\mathscr{E}^{J}\right|$, and $\mathscr{E}^{J}$ is a partition of $E^{J}$ into pairs, so $|T|=\left|\mathscr{E}^{J}\right|=\left|E^{J}\right| / 2$.

(ii) In view of Proposition 1 (i), any stable set $T$ in $H$ picks at most one node of each pair $\{e, \bar{e}\} \in \mathscr{E}^{J}$, hence $|T| \leq\left|E^{J}\right| / 2$. Asking $|T|=\left|E^{J}\right| / 2$ implies that exactly one node is picked from each pair $\{e, \bar{e}\} \in \mathscr{E}^{J}$, hence insertion $T$ is complete.

Proof (of Theorem 1) In view of Proposition 2, we just need to show that any stable set $T$ of size $\left|E^{J}\right| / 2$ corresponds to a positive acyclic insertion. Assume the contrary, i.e., insertion $T$ is positive cyclic, and let $Z^{\prime}$ be a positive cycle in $\left(V, A^{J} \cup T, d\right)$. By the SCP there exists a short positive cycle $Z$ with $Z \cap E^{J} \subseteq Z^{\prime} \cap E^{J} \subseteq T$ and $\left|Z \cap E^{J}\right|=2$, so $\{e, f\}=Z \cap E^{J}$ for some $e \in E^{J-}, f \in E^{J+}$. Then $\{e, f\}$ is a positive cyclic insertion, hence $\{e, f\} \in U$ contradicting the stability of $T$ in $H$.

Proof (of Theorem 2) We show that i) $\Phi(\{g\})$ is stable in $H$, ii) $T_{g}$ is stable in $H$, iii) $T_{g}$ is complete.

i) By definition of the closure and the bipartition of $H, \Phi(\{g\}) \subseteq E^{J+}$ if $g \in E^{J+}$ and $\Phi(\{g\}) \subseteq E^{J-}$ if $g \in E^{J-}$. $E^{J+}$ and $E^{J-}$ are the partitions of the bipartite conflict graph $H$ by Proposition 1 ii), so $\Phi(\{g\})$ is stable.

ii) $T_{g}$ is stable in $H$. Assuming the contrary, there exists some pair $e, f \in T_{g}$ so that $\{e, f\} \in U$. By i) $\Phi(\{g\})$ is stable in $H$. $T^{S}$ is a feasible insertion, hence it is stable in $H$ by Theorem 1 , then $T^{S} \backslash[\Phi(\{g\})]$ is stable in $H$. Therefore, we can assume that $e \in \Phi(\{g\})$ and $f \in T^{S} \backslash[\Phi(\{g\})]$. But by definition $e \rightsquigarrow \bar{f}$, so $\bar{f} \in \Phi(\{g\})$, contradicting $f \in T^{S} \backslash[\Phi(\{g\})]$.

iii) As $T^{S}$ is complete and by construction of $\Phi(\{g\})$ and $T^{S} \backslash[\Phi(\{g\})],\{e, \bar{e}\} \cap$ $T_{g} \neq \emptyset$ for all pairs $\{e, \bar{e}\} \in \mathscr{E} J$ implying completeness of $T_{g}$.

\subsection{Detailed results}

The detailed results of the JILS are recorded in Table 5 to 11 . All values are rounded to the nearest integer. 
Table 5: Objective values obtained with JILS for the Taillard benchmark instances in the JS with makespan objective. For each instance, the four columns display the average results (over the five runs) after 60, 300, and 1800 seconds and the best results after 1800 seconds. The instances are grouped according to their size $n \times m$, where $n$ is the number of jobs and $m$ the number of machines.

\begin{tabular}{|c|c|c|c|c|c|c|c|c|c|}
\hline \multirow{2}{*}{$\begin{array}{l}\text { objective } \\
\text { time }\end{array}$} & \multicolumn{4}{|c|}{ makespan } & & \multicolumn{4}{|c|}{ makespan } \\
\hline & 60 & 300 & 1800 & best & & 60 & 300 & 1800 & best \\
\hline $15 \times 15$ & & & & & & & & & \\
\hline ta01 & 1239 & 1233 & 1232 & 1231 & ta26 & 1671 & 1661 & 1659 & 1654 \\
\hline ta02 & 1256 & 1248 & 1245 & 1244 & ta27 & 1711 & 1697 & 1694 & 1687 \\
\hline ta03 & 1225 & 1224 & 1223 & 1221 & ta28 & 1633 & 1626 & 1624 & 1620 \\
\hline ta04 & 1182 & 1181 & 1177 & 1175 & ta29 & 1643 & 1637 & 1633 & 1629 \\
\hline ta05 & 1234 & 1233 & 1232 & 1231 & ta30 & 1623 & 1608 & 1603 & 1590 \\
\hline ta06 & 1246 & 1244 & 1242 & 1240 & $30 \times 15$ & & & & \\
\hline ta07 & 1228 & 1228 & 1228 & 1228 & ta31 & 1788 & 1767 & 1766 & 1764 \\
\hline ta08 & 1224 & 1219 & 1218 & 1217 & ta32 & 1861 & 1832 & 1829 & 1821 \\
\hline ta09 & 1287 & 1286 & 1283 & 1281 & ta33 & 1858 & 1823 & 1818 & 1808 \\
\hline ta 10 & 1262 & 1249 & 1245 & 1244 & ta34 & 1881 & 1845 & 1843 & 1837 \\
\hline $20 \times 15$ & & & & & ta35 & 2007 & 2007 & 2007 & 2007 \\
\hline ta11 & 1387 & 1377 & 1375 & 1369 & ta36 & 1849 & 1834 & 1828 & 1820 \\
\hline ta 12 & 1381 & 1377 & 1375 & 1373 & ta37 & 1830 & 1803 & 1800 & 1795 \\
\hline ta 13 & 1364 & 1355 & 1355 & 1352 & ta38 & 1715 & 1693 & 1688 & 1685 \\
\hline ta14 & 1352 & 1346 & 1345 & 1345 & ta39 & 1816 & 1806 & 1805 & 1797 \\
\hline ta15 & 1366 & 1357 & 1353 & 1343 & ta40 & 1730 & 1707 & 1706 & 1697 \\
\hline ta 16 & 1378 & 1371 & 1369 & 1362 & $30 \times 20$ & & & & \\
\hline ta 17 & 1489 & 1483 & 1476 & 1470 & ta41 & 2110 & 2068 & 2060 & 2053 \\
\hline ta 18 & 1429 & 1418 & 1418 & 1412 & $\mathrm{ta} 42$ & 2033 & 1990 & 1982 & 1974 \\
\hline ta 19 & 1358 & 1349 & 1345 & 1344 & $\mathrm{ta} 43$ & 1943 & 1900 & 1897 & 1889 \\
\hline ta20 & 1376 & 1367 & 1363 & 1356 & ta44 & 2061 & 2029 & 2017 & 2006 \\
\hline $20 \times 20$ & & & & & ta45 & 2068 & 2031 & 2020 & 2007 \\
\hline ta21 & 1673 & 1664 & 1660 & 1657 & ta46 & 2108 & 2072 & 2069 & 2060 \\
\hline ta22 & 1636 & 1624 & 1619 & 1609 & $\mathrm{ta} 47$ & 1983 & 1952 & 1942 & 1932 \\
\hline ta23 & 1579 & 1570 & 1566 & 1560 & ta48 & 2047 & 1993 & 1987 & 1975 \\
\hline ta24 & 1671 & 1663 & 1661 & 1658 & ta49 & 2054 & 2018 & 2011 & 2000 \\
\hline ta25 & 1630 & 1615 & 1604 & 1599 & $\operatorname{ta} 50$ & 2021 & 1977 & 1966 & 1947 \\
\hline
\end{tabular}


Table 6: Objective values obtained with JILS in the JS with makespan, total flow time, and total squared flow time objective. Results for the total squared flow time objective are provided in units of 1000 .

\begin{tabular}{|c|c|c|c|c|c|c|c|c|c|c|c|c|}
\hline \multirow{2}{*}{$\begin{array}{l}\text { objective } \\
\text { time }\end{array}$} & \multicolumn{4}{|c|}{ makespan } & \multicolumn{4}{|c|}{ total flow time } & \multicolumn{4}{|c|}{ total squared flow time } \\
\hline & 60 & 300 & 1800 & best & 60 & 300 & 1800 & best & 60 & 300 & 1800 & best \\
\hline \multicolumn{13}{|l|}{$10 \times 5$} \\
\hline $\mathrm{la} 01$ & 666 & 666 & 666 & 666 & 4834 & 4832 & 4832 & 4832 & 2602 & 2599 & 2599 & 2599 \\
\hline la02 & 655 & 655 & 655 & 655 & 4481 & 4468 & 4463 & 4459 & 2298 & 2297 & 2296 & 2296 \\
\hline la03 & 597 & 597 & 597 & 597 & 4152 & 4151 & 4151 & 4151 & 1956 & 1956 & 1956 & 1956 \\
\hline la04 & 590 & 590 & 590 & 590 & 4259 & 4259 & 4259 & 4259 & 2060 & 2060 & 2060 & 2060 \\
\hline la05 & 593 & 593 & 593 & 593 & 4074 & 4072 & 4072 & 4072 & 1862 & 1862 & 1862 & 1862 \\
\hline \multicolumn{13}{|l|}{$15 \times 5$} \\
\hline la06 & 926 & 926 & 926 & 926 & 8725 & 8649 & 8629 & 8626 & 6038 & 5967 & 5953 & 5941 \\
\hline la07 & 890 & 890 & 890 & 890 & 8166 & 8122 & 8081 & 8069 & 5364 & 5287 & 5252 & 5240 \\
\hline la08 & 863 & 863 & 863 & 863 & 8130 & 7989 & 7958 & 7946 & 5279 & 5216 & 5188 & 5093 \\
\hline la09 & 951 & 951 & 951 & 951 & 9215 & 9069 & 9050 & 9043 & 6669 & 6571 & 6553 & 6520 \\
\hline la10 & 958 & 958 & 958 & 958 & 9068 & 8885 & 8866 & 8818 & 6461 & 6399 & 6348 & 6309 \\
\hline \multicolumn{13}{|l|}{$20 \times 5$} \\
\hline la11 & 1222 & 1222 & 1222 & 1222 & 14530 & 14249 & 14053 & 13936 & 13060 & 12727 & 12505 & 12441 \\
\hline la12 & 1039 & 1039 & 1039 & 1039 & 12454 & 12031 & 11870 & 11759 & 9709 & 9489 & 9420 & 9265 \\
\hline la13 & 1150 & 1150 & 1150 & 1150 & 13945 & 13564 & 13410 & 13358 & 11864 & 11544 & 11350 & 11254 \\
\hline la14 & 1292 & 1292 & 1292 & 1292 & 15072 & 14781 & 14644 & 14622 & 13980 & 13783 & 13596 & 13274 \\
\hline la15 & 1207 & 1207 & 1207 & 1207 & 15007 & 14544 & 14316 & 14216 & 13368 & 13033 & 12884 & 12754 \\
\hline \multicolumn{13}{|l|}{$10 \times 10$} \\
\hline la16 & 945 & 945 & 945 & 945 & 7383 & 7376 & 7376 & 7376 & 5771 & 5771 & 5771 & 5771 \\
\hline la17 & 784 & 784 & 784 & 784 & 6565 & 6547 & 6537 & 6537 & 4594 & 4565 & 4557 & 4556 \\
\hline la18 & 848 & 848 & 848 & 848 & 6993 & 6971 & 6970 & 6970 & 5153 & 5132 & 5127 & 5117 \\
\hline la19 & 842 & 842 & 842 & 842 & 7248 & 7227 & 7217 & 7217 & 5351 & 5335 & 5335 & 5335 \\
\hline la20 & 902 & 902 & 902 & 902 & 7403 & 7371 & 7354 & 7345 & 5840 & 5808 & 5792 & 5764 \\
\hline orb01 & 1061 & 1059 & 1059 & 1059 & 8187 & 8093 & 8044 & 8023 & 6980 & 6881 & 6858 & 6856 \\
\hline orb02 & 888 & 888 & 888 & 888 & 7344 & 7333 & 7316 & 7308 & 5616 & 5611 & 5606 & 5606 \\
\hline orb03 & 1005 & 1005 & 1005 & 1005 & 8202 & 8085 & 8056 & 8032 & 7072 & 6898 & 6865 & 6865 \\
\hline orb04 & 1005 & 1005 & 1005 & 1005 & 7897 & 7893 & 7893 & 7893 & 6744 & 6744 & 6744 & 6744 \\
\hline orb05 & 889 & 889 & 888 & 887 & 6989 & 6983 & 6983 & 6983 & 5153 & 5148 & 5141 & 5135 \\
\hline orb06 & 1012 & 1010 & 1010 & 1010 & 8179 & 8137 & 8130 & 8127 & 7065 & 7003 & 6984 & 6982 \\
\hline orb07 & 397 & 397 & 397 & 397 & 3293 & 3259 & 3257 & 3257 & 1146 & 1111 & 1108 & 1108 \\
\hline orb08 & 899 & 899 & 899 & 899 & 6981 & 6951 & 6944 & 6931 & 5276 & 5234 & 5209 & 5198 \\
\hline orb09 & 934 & 934 & 934 & 934 & 7452 & 7433 & 7433 & 7433 & 6068 & 6040 & 6016 & 6008 \\
\hline orb10 & 944 & 944 & 944 & 944 & 7905 & 7850 & 7846 & 7846 & 6461 & 6365 & 6364 & 6364 \\
\hline \multicolumn{13}{|l|}{$15 \times 10$} \\
\hline la21 & 1051 & 1048 & 1047 & 1046 & 13024 & 12473 & 12353 & 12208 & 11613 & 11265 & 11207 & 11010 \\
\hline la22 & 932 & 932 & 929 & 927 & 12457 & 11919 & 11825 & 11720 & 10291 & 9955 & 9798 & 9711 \\
\hline la23 & 1032 & 1032 & 1032 & 10 & 13063 & 12822 & 12692 & 12650 & 11606 & 11329 & 11210 & 11112 \\
\hline la24 & 943 & 940 & 938 & 935 & 12686 & 12137 & 12058 & 11936 & 10460 & 10279 & 10153 & 9846 \\
\hline la25 & 978 & 977 & 977 & 977 & 12383 & 11952 & 11868 & 11818 & 10385 & 10076 & 9911 & 9802 \\
\hline \multicolumn{13}{|l|}{$20 \times 10$} \\
\hline la26 & 1218 & 1218 & 1218 & 1218 & 23842 & 21428 & 20271 & 19614 & 21671 & 20942 & 20477 & 19838 \\
\hline la27 & 1240 & 1237 & 1235 & 1235 & 25710 & 22158 & 20232 & 19939 & 22908 & 22498 & 21674 & 21517 \\
\hline la28 & 1216 & 1216 & 1216 & 1216 & 24534 & 22262 & 20425 & 20000 & 21573 & 21106 & 20696 & 20237 \\
\hline la29 & 1173 & 1170 & 1166 & 1164 & 22521 & 20641 & 18657 & 17992 & 19357 & 18533 & 18006 & 17731 \\
\hline la30 & 1355 & 1355 & 1355 & 1355 & 23328 & 21020 & 19486 & 19249 & 22579 & 21839 & 21014 & 20784 \\
\hline \multicolumn{13}{|l|}{$30 \times 10$} \\
\hline la31 & 1784 & 1784 & 1784 & 1784 & 57420 & 54393 & 49893 & 45168 & 55463 & 54369 & 53638 & 51998 \\
\hline la32 & 1850 & 1850 & 1850 & 1850 & 64989 & 60314 & 55884 & 51938 & 62797 & 61517 & 60394 & 58639 \\
\hline la33 & 1719 & 1719 & 1719 & 1719 & 61882 & 56276 & 49519 & 45454 & 51619 & 50801 & 49765 & 48622 \\
\hline la34 & 1721 & 1721 & 1721 & 1721 & 63195 & 58745 & 54917 & 49862 & 55660 & 54501 & 53440 & 52042 \\
\hline la35 & 1888 & 1888 & 1888 & 1888 & 60661 & 57369 & 51645 & 46655 & 57721 & 56895 & 56239 & 55458 \\
\hline \multicolumn{13}{|l|}{$15 \times 15$} \\
\hline la36 & 1272 & 1268 & 1268 & 1268 & 18871 & 17428 & 16806 & 16620 & 20474 & 19873 & 19607 & 19331 \\
\hline la37 & 1410 & 1405 & 1403 & 1401 & 19467 & 18404 & 17765 & 17670 & 22479 & 21776 & 21647 & 21321 \\
\hline la38 & 1201 & 1199 & 1196 & 1196 & 18238 & 16524 & 16040 & 15717 & 17309 & 16899 & 16669 & 16233 \\
\hline la39 & 1234 & 1234 & 1234 & 1233 & 18345 & 16589 & 16198 & 15922 & 18193 & 17866 & 17460 & 16985 \\
\hline la40 & 1229 & 1227 & 1226 & 1226 & 18712 & 16995 & 16466 & 16069 & 18378 & 17886 & 17537 & 17234 \\
\hline
\end{tabular}


Table 7: Objective values obtained with JILS in the JS with total tardiness and total weighted tardiness objective.

\begin{tabular}{|c|c|c|c|c|c|c|c|c|}
\hline \multirow{2}{*}{$\begin{array}{l}\text { objective } \\
\text { time }\end{array}$} & \multicolumn{4}{|c|}{ total tardiness } & \multicolumn{4}{|c|}{ total weighted tardiness } \\
\hline & 60 & 300 & 1800 & best & 60 & 300 & 1800 & best \\
\hline \multicolumn{9}{|l|}{$10 \times 5$} \\
\hline la01 & 1194 & 1194 & 1194 & 1194 & 2299 & 2299 & 2299 & 2299 \\
\hline la02 & 1076 & 1071 & 1065 & 1065 & 1762 & 1762 & 1762 & 1762 \\
\hline la03 & 1076 & 1076 & 1076 & 1076 & 1951 & 1951 & 1951 & 1951 \\
\hline la04 & 1096 & 1096 & 1096 & 1096 & 1917 & 1917 & 1917 & 1917 \\
\hline la05 & 1165 & 1164 & 1164 & 1164 & 1878 & 1878 & 1878 & 1878 \\
\hline \multicolumn{9}{|l|}{$15 \times 5$} \\
\hline la06 & 3541 & 3495 & 3491 & 3488 & 6026 & 5883 & 5813 & 5810 \\
\hline la07 & 3359 & 3309 & 3282 & 3272 & 5952 & 5855 & 5769 & 5765 \\
\hline la08 & 3105 & 3083 & 3056 & 3027 & 5532 & 5475 & 5475 & 5475 \\
\hline la09 & 3699 & 3598 & 3567 & 3567 & 5811 & 5608 & 5608 & 5608 \\
\hline la10 & 3805 & 3688 & 3655 & 3604 & 6795 & 6687 & 6618 & 6618 \\
\hline \multicolumn{9}{|l|}{$20 \times 5$} \\
\hline la11 & 7301 & 7136 & 7032 & 6929 & 12474 & 12355 & 12043 & 11885 \\
\hline la12 & 5801 & 5750 & 5702 & 5668 & 11009 & 10862 & 10737 & 10542 \\
\hline la13 & 6756 & 6692 & 6659 & 6618 & 11878 & 11652 & 11539 & 11475 \\
\hline la14 & 7838 & 7771 & 7758 & 7675 & 13453 & 13345 & 13244 & 13107 \\
\hline la15 & 7306 & 7166 & 7109 & 7089 & 13016 & 12668 & 12595 & 12354 \\
\hline \multicolumn{9}{|l|}{$10 \times 10$} \\
\hline la16 & 616 & 612 & 612 & 612 & 1169 & 1169 & 1169 & 1169 \\
\hline la17 & 711 & 694 & 694 & 694 & 927 & 899 & 899 & 899 \\
\hline la18 & 484 & 484 & 484 & 484 & 935 & 929 & 929 & 929 \\
\hline la19 & 486 & 486 & 486 & 486 & 973 & 949 & 948 & 948 \\
\hline la20 & 555 & 546 & 538 & 536 & 831 & 820 & 805 & 805 \\
\hline orb01 & 1414 & 1262 & 1246 & 1246 & 2625 & 2578 & 2568 & 2568 \\
\hline orb02 & 707 & 693 & 676 & 656 & 1423 & 1416 & 1408 & 1408 \\
\hline orb03 & 1381 & 1320 & 1271 & 1271 & 2186 & 2112 & 2111 & 2111 \\
\hline orb04 & 829 & 823 & 823 & 823 & 1635 & 1623 & 1623 & 1623 \\
\hline orb05 & 825 & 809 & 806 & 806 & 1742 & 1688 & 1667 & 1667 \\
\hline orb06 & 982 & 973 & 959 & 959 & 1821 & 1790 & 1790 & 1790 \\
\hline orb07 & 286 & 284 & 284 & 284 & 591 & 590 & 590 & 590 \\
\hline orb08 & 1240 & 1237 & 1216 & 1211 & 2534 & 2466 & 2436 & 2429 \\
\hline orb09 & 857 & 856 & 844 & 844 & 1316 & 1316 & 1316 & 1316 \\
\hline orb10 & 891 & 863 & 863 & 863 & 1795 & 1748 & 1709 & 1679 \\
\hline \multicolumn{9}{|l|}{$15 \times 10$} \\
\hline la21 & 2263 & 2163 & 2120 & 2098 & 3974 & 3670 & 3608 & 3560 \\
\hline la22 & 2599 & 2448 & 2392 & 2264 & 4909 & 4638 & 4579 & 4356 \\
\hline la23 & 2522 & 2359 & 2267 & 2244 & 4133 & 4073 & 3922 & 3777 \\
\hline la24 & 2356 & 2262 & 2148 & 2113 & 3945 & 3804 & 3759 & 3579 \\
\hline la25 & 2297 & 2186 & 2162 & 2092 & 3862 & 3586 & 3527 & 3313 \\
\hline \multicolumn{9}{|l|}{$20 \times 10$} \\
\hline la26 & 6082 & 5766 & 5540 & 5417 & 10756 & 10607 & 10074 & 9803 \\
\hline la27 & 6023 & 5927 & 5658 & 5480 & 10158 & 9725 & 9570 & 9226 \\
\hline la28 & 6088 & 5888 & 5566 & 5302 & 10572 & 10208 & 9810 & 9606 \\
\hline la29 & 5412 & 5132 & 4867 & 4637 & 10577 & 10377 & 10041 & 9556 \\
\hline la30 & 5672 & 5509 & 5243 & 5150 & 9864 & 9594 & 9268 & 8981 \\
\hline \multicolumn{9}{|l|}{$30 \times 10$} \\
\hline la31 & 17329 & 17065 & 16521 & 16158 & 31072 & 30809 & 30200 & 29039 \\
\hline la32 & 18077 & 17861 & 17265 & 16846 & 33380 & 32851 & 32544 & 32258 \\
\hline la33 & 16440 & 16253 & 15746 & 15554 & 28996 & 28441 & 28098 & 26931 \\
\hline la34 & 16576 & 16323 & 16010 & 15465 & 30128 & 29461 & 28806 & 28352 \\
\hline la35 & 17481 & 17214 & 16636 & 16397 & 32288 & 31945 & 31259 & 30730 \\
\hline \multicolumn{9}{|l|}{$15 \times 15$} \\
\hline la36 & 1988 & 1842 & 1797 & 1792 & 3578 & 3286 & 3196 & 2981 \\
\hline la37 & 2128 & 1884 & 1753 & 1565 & 3053 & 2726 & 2608 & 2290 \\
\hline la38 & 1484 & 1348 & 1278 & 1101 & 2795 & 2496 & 2400 & 2159 \\
\hline la39 & 1200 & 1040 & 971 & 907 & 2341 & 1912 & 1820 & 1676 \\
\hline la40 & 1530 & 1318 & 1148 & 1107 & 2811 & 2561 & 2347 & 2159 \\
\hline
\end{tabular}


Table 8: Objective values obtained with JILS in the JSS with makespan, total flow time, and total squared flow time objective. Results for the total squared flow time objective are provided in units of 1000 .

\begin{tabular}{|c|c|c|c|c|c|c|c|c|c|c|c|c|}
\hline \multirow{2}{*}{$\begin{array}{l}\text { objective } \\
\text { time }\end{array}$} & \multicolumn{4}{|c|}{ makespan } & \multicolumn{4}{|c|}{ total flow time } & \multicolumn{4}{|c|}{ total squared flow time } \\
\hline & 60 & 300 & 1800 & best & 60 & 300 & 1800 & best & 60 & 300 & 1800 & best \\
\hline \multicolumn{13}{|l|}{$10 \times 5$} \\
\hline t2-ps01 & 798 & 798 & 798 & 798 & 6057 & 6053 & 6050 & 6050 & 4083 & 4060 & 4053 & 4053 \\
\hline $\mathrm{t} 2-\mathrm{ps} 02$ & 784 & 784 & 784 & 784 & 5509 & 5505 & 5505 & 5505 & 3479 & 3479 & 3479 & 3479 \\
\hline $\mathrm{t} 2-\mathrm{ps} 03$ & 749 & 749 & 749 & 749 & 5113 & 5092 & 5092 & 5092 & 2988 & 2973 & 2973 & 2973 \\
\hline t2-ps04 & 732 & 730 & 730 & 730 & 5521 & 5485 & 5478 & 5478 & 3406 & 3375 & 3375 & 3375 \\
\hline t2-ps05 & 691 & 691 & 691 & 691 & 5050 & 5041 & 5041 & 5041 & 2885 & 2876 & 2874 & 2874 \\
\hline \multicolumn{13}{|l|}{$15 \times 5$} \\
\hline t2-ps06 & 1026 & 1026 & 1026 & 1025 & 11017 & 10773 & 10706 & 10608 & 9035 & 8870 & 8685 & 8610 \\
\hline $\mathrm{t} 2-\mathrm{ps} 07$ & 970 & 970 & 970 & 970 & 10332 & 10187 & 10144 & 10039 & 8188 & 7982 & 7912 & 7851 \\
\hline t2-ps08 & 975 & 967 & 965 & 963 & 10428 & 10255 & 10193 & 10140 & 8403 & 8121 & 8019 & 7816 \\
\hline t2-ps09 & 1060 & 1060 & 1060 & 1060 & 11303 & 11045 & 10879 & 10835 & 9936 & 9503 & 9346 & 9329 \\
\hline $\mathrm{t} 2-\mathrm{ps} 10$ & 1018 & 1018 & 1018 & 1018 & 11040 & 10924 & 10828 & 10785 & 9311 & 9027 & 8950 & 8800 \\
\hline \multicolumn{13}{|l|}{$20 \times 5$} \\
\hline t2-ps11 & 1506 & 1504 & 1489 & 1460 & 21185 & 20506 & 20180 & 19666 & 28742 & 27633 & 25810 & 24650 \\
\hline $\mathrm{t} 2-\mathrm{ps} 12$ & 1334 & 1333 & 1324 & 1319 & 19682 & 19176 & 18783 & 18534 & 22397 & 22069 & 21055 & 20285 \\
\hline $\mathrm{t} 2-\mathrm{ps} 13$ & 1437 & 1437 & 1434 & 1430 & 21315 & 21044 & 20666 & 20162 & 26512 & 25750 & 24805 & 24011 \\
\hline t2-ps 14 & 1489 & 1489 & 1480 & 1469 & 21738 & 21378 & 21015 & 20595 & 28982 & 27816 & 26097 & 24860 \\
\hline $\mathrm{t} 2-\mathrm{ps} 15$ & 1531 & 1526 & 1524 & 1518 & 21977 & 21438 & 21065 & 20657 & 29371 & 27908 & 26871 & 26338 \\
\hline \multicolumn{13}{|l|}{$15 \times 10$} \\
\hline t2-LA21sdst & 1279 & 1273 & 1273 & 1273 & 15529 & 15233 & 15116 & 15009 & 17543 & 17137 & 16993 & 16644 \\
\hline t2-LA24sdst & 1163 & 1157 & 1155 & 1154 & 15317 & 14899 & 14780 & 14630 & 16538 & 15854 & 15658 & 15380 \\
\hline t2-LA25sdst & 1198 & 1192 & 1188 & 1184 & 14964 & 14693 & 14590 & 14441 & 16079 & 15713 & 15485 & 15234 \\
\hline \multicolumn{13}{|l|}{$20 \times 10$} \\
\hline t2-LA27sdst & 1826 & 1812 & 1791 & 1767 & 30471 & 30005 & 29445 & 28705 & 52949 & 51839 & 50598 & 47885 \\
\hline t2-LA29sdst & 1720 & 1698 & 1691 & 1678 & 28663 & 28384 & 27571 & 27231 & 46925 & 46267 & 44236 & 42311 \\
\hline \multicolumn{13}{|l|}{$15 \times 15$} \\
\hline t2-LA38sdst & 1471 & 1463 & 1456 & 1447 & 19283 & 18853 & 18733 & 18521 & 25947 & 25412 & 25024 & 24265 \\
\hline t2-LA40sdst & 1499 & 1492 & 1488 & 1480 & 20051 & 19615 & 19458 & 19267 & 28114 & 26915 & 26464 & 26002 \\
\hline \multicolumn{13}{|l|}{$20 \times 15$} \\
\hline t2-ABZ7sdst & 1359 & 1305 & 1299 & 1287 & 23536 & 23376 & 22916 & 22446 & 30374 & 29786 & 29566 & 28321 \\
\hline t2-ABZ8sdst & 1367 & 1343 & 1329 & 1307 & 23754 & 23500 & 23173 & 22630 & 31503 & 31185 & 30423 & 28890 \\
\hline t2-ABZ9sdst & 1343 & 1306 & 1296 & 1286 & 23366 & 23176 & 22753 & 22388 & 30955 & 30445 & 30331 & 29121 \\
\hline
\end{tabular}


Table 9: Objective values obtained with JILS in the JSS with total tardiness and total weighted tardiness objective.

\begin{tabular}{|c|c|c|c|c|c|c|c|c|}
\hline \multirow{2}{*}{$\begin{array}{l}\text { objective } \\
\text { time }\end{array}$} & \multicolumn{4}{|c|}{ total tardiness } & \multicolumn{4}{|c|}{ total weighted tardiness } \\
\hline & 60 & 300 & 1800 & best & 60 & 300 & 1800 & best \\
\hline \multicolumn{9}{|l|}{$10 \times 5$} \\
\hline t2-ps01 & 1659 & 1631 & 1623 & 1623 & 2868 & 2852 & 2852 & 2852 \\
\hline t2-ps02 & 1395 & 1395 & 1395 & 1395 & 2312 & 2301 & 2301 & 2301 \\
\hline t2-ps03 & 1376 & 1361 & 1361 & 1361 & 2689 & 2677 & 2677 & 2677 \\
\hline t2-ps04 & 1594 & 1583 & 1583 & 1583 & 2538 & 2538 & 2538 & 2538 \\
\hline t2-ps05 & 1546 & 1545 & 1545 & 1545 & 2636 & 2602 & 2570 & 2570 \\
\hline \multicolumn{9}{|l|}{$15 \times 5$} \\
\hline t2-ps06 & 4531 & 4441 & 4379 & 4361 & 7727 & 7493 & 7359 & 7359 \\
\hline t2-ps07 & 4406 & 4262 & 4180 & 4143 & 7587 & 7418 & 7407 & 7358 \\
\hline t2-ps08 & 4378 & 4164 & 4083 & 4062 & 8153 & 7823 & 7627 & 7524 \\
\hline t2-ps09 & 4391 & 4215 & 4155 & 4147 & 7619 & 7411 & 7314 & 7303 \\
\hline t2-ps 10 & 4602 & 4473 & 4305 & 4237 & 8330 & 7975 & 7872 & 7837 \\
\hline \multicolumn{9}{|l|}{$20 \times 5$} \\
\hline t2-ps11 & 12884 & 12446 & 11947 & 11561 & 22873 & 22506 & 22110 & 21365 \\
\hline t2-ps 12 & 12378 & 11907 & 11510 & 10918 & 21935 & 21674 & 21171 & 20937 \\
\hline t2-ps13 & 12766 & 12565 & 12224 & 11904 & 22068 & 21684 & 21364 & 20929 \\
\hline t2-ps 14 & 13197 & 12937 & 12511 & 12365 & 24423 & 23776 & 23140 & 22446 \\
\hline t2-ps15 & 12977 & 12586 & 12179 & 12023 & 24099 & 23200 & 22721 & 22127 \\
\hline \multicolumn{9}{|l|}{$15 \times 10$} \\
\hline t2-LA21sdst & 2961 & 2772 & 2674 & 2609 & 4851 & 4597 & 4454 & 4347 \\
\hline $\mathrm{t} 2-\mathrm{LA} 24 \mathrm{sdst}$ & 2911 & 2661 & 2490 & 2300 & 5457 & 5114 & 4920 & 4492 \\
\hline t2-LA25sdst & 3181 & 2918 & 2831 & 2763 & 5645 & 4880 & 4683 & 4454 \\
\hline \multicolumn{9}{|l|}{$20 \times 10$} \\
\hline t2-LA27sdst & 12939 & 12566 & 12022 & 11592 & 23105 & 22605 & 21631 & 20368 \\
\hline t2-LA29sdst & 12723 & 12449 & 11648 & 11360 & 23395 & 22973 & 22149 & 21493 \\
\hline \multicolumn{9}{|l|}{$15 \times 15$} \\
\hline t2-LA38sdst & 1925 & 1689 & 1445 & 1282 & 3642 & 3118 & 2632 & 2417 \\
\hline t2-LA40sdst & 2421 & 2100 & 1828 & 1637 & 4651 & 3844 & 2912 & 2137 \\
\hline \multicolumn{9}{|l|}{$20 \times 15$} \\
\hline t2-ABZ7sdst & 12019 & 11716 & 11175 & 10804 & 20452 & 20210 & 19336 & 18378 \\
\hline t2-ABZ8sdst & 11613 & 11293 & 10818 & 10331 & 20453 & 19986 & 19168 & 18759 \\
\hline t2-ABZ9sdst & 11291 & 11013 & 10664 & 10473 & 19877 & 19470 & 19091 & 18710 \\
\hline
\end{tabular}


Table 10: Objective values obtained with JILS in the BJS with makespan, total flow time, and total squared flow time objective. Results for the total squared flow time objective are provided in units of 1000 .

\begin{tabular}{|c|c|c|c|c|c|c|c|c|c|c|c|c|}
\hline \multirow{2}{*}{$\begin{array}{l}\text { objective } \\
\text { time }\end{array}$} & \multicolumn{4}{|c|}{ makespan } & \multicolumn{4}{|c|}{ total flow time } & \multicolumn{4}{|c|}{ total squared flow time } \\
\hline & 60 & 300 & 1800 & best & 60 & 300 & 1800 & best & 60 & 300 & 1800 & best \\
\hline \multicolumn{13}{|l|}{$10 \times 5$} \\
\hline la01 & 793 & 793 & 793 & 793 & 5174 & 5152 & 5152 & 5152 & 3123 & 3123 & 3123 & 3123 \\
\hline la02 & 793 & 793 & 793 & 793 & 4937 & 4937 & 4937 & 4937 & 2982 & 2982 & 2982 & 2982 \\
\hline la03 & 715 & 715 & 715 & 715 & 4605 & 4605 & 4605 & 4605 & 2574 & 2574 & 2574 & 2574 \\
\hline la04 & 743 & 743 & 743 & 743 & 4781 & 4773 & 4764 & 4764 & 2663 & 2663 & 2663 & 2663 \\
\hline la05 & 664 & 664 & 664 & 664 & 4447 & 4447 & 4447 & 4447 & 2309 & 2309 & 2309 & 2309 \\
\hline \multicolumn{13}{|l|}{$15 \times 5$} \\
\hline la06 & 1099 & 1085 & 1066 & 1060 & 10035 & 9860 & 9715 & 9683 & 8113 & 7963 & 7769 & 7689 \\
\hline la07 & 1054 & 1038 & 1025 & 1016 & 9349 & 9264 & 9136 & 9083 & 7141 & 6920 & 6715 & 6684 \\
\hline la08 & 1091 & 1064 & 1044 & 1040 & 9338 & 9138 & 9042 & 9042 & 7503 & 7091 & 6722 & 6604 \\
\hline la09 & 1181 & 1167 & 1145 & 1141 & 10590 & 10312 & 10237 & 10185 & 9273 & 8958 & 8690 & 8613 \\
\hline la10 & 1143 & 1120 & 1111 & 1096 & 9966 & 9840 & 9758 & 9735 & 8376 & 8166 & 7986 & 7920 \\
\hline \multicolumn{13}{|l|}{$20 \times 5$} \\
\hline la11 & 1497 & 1478 & 1461 & 1446 & 16778 & 16258 & 16073 & 15787 & 17973 & 17811 & 17146 & 16444 \\
\hline la12 & 1295 & 1273 & 1254 & 1239 & 14242 & 13897 & 13634 & 13464 & 13808 & 13292 & 12843 & 12098 \\
\hline la13 & 1461 & 1428 & 1399 & 1361 & 16083 & 15791 & 15379 & 15243 & 17090 & 16486 & 16189 & 15912 \\
\hline la14 & 1512 & 1498 & 1473 & 1464 & 16869 & 16569 & 16295 & 16180 & 18376 & 18247 & 17796 & 17521 \\
\hline la15 & 1533 & 1512 & 1492 & 1477 & 17072 & 16772 & 16575 & 16315 & 20066 & 18935 & 18517 & 18264 \\
\hline \multicolumn{13}{|l|}{$10 \times 10$} \\
\hline la16 & 1078 & 1066 & 1060 & 1060 & 8222 & 8166 & 8123 & 8109 & 7378 & 7218 & 7187 & 7187 \\
\hline la17 & 932 & 930 & 929 & 929 & 7385 & 7224 & 7159 & 7159 & 5677 & 5581 & 5570 & 5529 \\
\hline la18 & 1039 & 1033 & 1025 & 1025 & 7744 & 7652 & 7525 & 7525 & 6455 & 6366 & 6350 & 6350 \\
\hline la19 & 1065 & 1049 & 1045 & 1043 & 8216 & 8165 & 8086 & 8030 & 7204 & 7009 & 6919 & 6919 \\
\hline la20 & 1089 & 1067 & 1060 & 1060 & 8132 & 8020 & 7978 & 7951 & 7125 & 7062 & 7054 & 7054 \\
\hline \multicolumn{13}{|l|}{$15 \times 10$} \\
\hline la21 & 1486 & 1467 & 1425 & 1396 & 15312 & 14914 & 14665 & 14483 & 18985 & 16899 & 16601 & 15967 \\
\hline la22 & 1340 & 1328 & 1309 & 1293 & 14411 & 14117 & 13946 & 13479 & 15612 & 15110 & 14697 & 14490 \\
\hline la23 & 1481 & 1451 & 1426 & 1417 & 15682 & 15494 & 15229 & 15108 & 18426 & 17706 & 17268 & 16809 \\
\hline la24 & 1410 & 1390 & 1378 & 1366 & 15038 & 14698 & 14406 & 14209 & 17441 & 16124 & 15640 & 15107 \\
\hline la25 & 1413 & 1380 & 1330 & 1311 & 14466 & 14244 & 13937 & 13797 & 16543 & 15615 & 14918 & 14396 \\
\hline \multicolumn{13}{|l|}{$20 \times 10$} \\
\hline la26 & 1957 & 1908 & 1860 & 1848 & 25374 & 25124 & 24152 & 23437 & 38682 & 36714 & 35168 & 34597 \\
\hline la27 & 2031 & 1971 & 1934 & 1920 & 26045 & 25587 & 24884 & 24444 & 40974 & 39169 & 37271 & 36006 \\
\hline la28 & 1969 & 1926 & 1842 & 1829 & 25577 & 25208 & 24624 & 24023 & 39600 & 37149 & 36199 & 35492 \\
\hline la29 & 1799 & 1789 & 1749 & 1721 & 23578 & 23277 & 22398 & 22348 & 32782 & 30903 & 30211 & 29731 \\
\hline la30 & 1980 & 1927 & 1888 & 1869 & 24637 & 24292 & 23634 & 23190 & 37990 & 35864 & 35607 & 34754 \\
\hline \multicolumn{13}{|l|}{$30 \times 10$} \\
\hline la31 & 2841 & 2756 & 2694 & 2677 & 50773 & 49859 & 49444 & 48552 & 106731 & 104192 & 99488 & 96030 \\
\hline la32 & 3077 & 2979 & 2911 & 2860 & 55042 & 54271 & 53200 & 52092 & 126460 & 121433 & 116269 & 110294 \\
\hline la33 & 2747 & 2682 & 2615 & 2590 & 49697 & 49646 & 49163 & 47260 & 102757 & 98181 & 94674 & 91772 \\
\hline la34 & 2852 & 2773 & 2652 & 2619 & 51516 & 51301 & 50931 & 50251 & 111042 & 106008 & 101361 & 96075 \\
\hline la35 & 2887 & 2777 & 2717 & 2682 & 52149 & 51629 & 51095 & 50573 & 109471 & 103423 & 99284 & 97650 \\
\hline \multicolumn{13}{|l|}{$15 \times 15$} \\
\hline la36 & 1731 & 1719 & 1670 & 1639 & 19768 & 19588 & 19406 & 19059 & 28934 & 27923 & 27511 & 26621 \\
\hline la37 & 1871 & 1835 & 1804 & 1761 & 21588 & 21175 & 20790 & 20537 & 34209 & 31780 & 30889 & 29660 \\
\hline la38 & 1680 & 1635 & 1607 & 1593 & 18992 & 18854 & 18381 & 18199 & 25288 & 24826 & 23972 & 22757 \\
\hline la39 & 1749 & 1723 & 1659 & 1634 & 19415 & 19216 & 18889 & 18602 & 28135 & 26616 & 25605 & 24938 \\
\hline la 40 & 1749 & 1699 & 1645 & 1609 & 19527 & 19300 & 19006 & 18810 & 28666 & 28068 & 26502 & 24132 \\
\hline
\end{tabular}


Table 11: Objective values obtained with JILS in the BJS with total tardiness and total weighted tardiness objective.

\begin{tabular}{|c|c|c|c|c|c|c|c|c|}
\hline \multirow{2}{*}{$\begin{array}{l}\text { objective } \\
\text { time }\end{array}$} & \multicolumn{4}{|c|}{ total tardiness } & \multicolumn{4}{|c|}{ total weighted tardiness } \\
\hline & 60 & 300 & 1800 & best & 60 & 300 & 1800 & best \\
\hline \multicolumn{9}{|l|}{$10 \times 5$} \\
\hline la01 & 757 & 743 & 743 & 743 & 1486 & 1486 & 1486 & 1486 \\
\hline la02 & 705 & 700 & 700 & 700 & 1251 & 1251 & 1251 & 1251 \\
\hline la03 & 736 & 736 & 736 & 736 & 1461 & 1461 & 1461 & 1461 \\
\hline la04 & 720 & 716 & 716 & 716 & 1240 & 1236 & 1236 & 1236 \\
\hline la05 & 796 & 796 & 796 & 796 & 1245 & 1244 & 1244 & 1244 \\
\hline \multicolumn{9}{|l|}{$15 \times 5$} \\
\hline la06 & 3349 & 3192 & 3075 & 2994 & 5723 & 5569 & 5469 & 5339 \\
\hline la07 & 3088 & 3034 & 2946 & 2929 & 4920 & 4809 & 4727 & 4727 \\
\hline la08 & 3040 & 2878 & 2831 & 2813 & 4991 & 4956 & 4951 & 4949 \\
\hline la09 & 3379 & 3239 & 3127 & 3127 & 5800 & 5704 & 5685 & 5685 \\
\hline la10 & 3144 & 3060 & 2994 & 2984 & 5996 & 5950 & 5785 & 5514 \\
\hline \multicolumn{9}{|l|}{$20 \times 5$} \\
\hline la11 & 7289 & 7169 & 6873 & 6572 & 12943 & 12733 & 12077 & 11627 \\
\hline la12 & 5980 & 5909 & 5557 & 5417 & 11304 & 10618 & 10132 & 9789 \\
\hline la13 & 7300 & 6898 & 6566 & 6317 & 12650 & 11992 & 11483 & 11310 \\
\hline la14 & 7660 & 7533 & 7182 & 7089 & 12869 & 12486 & 12245 & 12129 \\
\hline la15 & 7739 & 7464 & 7109 & 6985 & 13550 & 12749 & 12321 & 12223 \\
\hline \multicolumn{9}{|l|}{$10 \times 10$} \\
\hline la16 & 63 & 25 & 22 & 20 & 96 & 56 & 44 & 40 \\
\hline la17 & 153 & 114 & 104 & 98 & 274 & 215 & 144 & 132 \\
\hline la18 & 94 & 53 & 11 & 3 & 91 & 71 & 24 & 6 \\
\hline la19 & 179 & 103 & 63 & 43 & 364 & 229 & 169 & 86 \\
\hline la20 & 36 & 0 & 0 & 0 & 42 & 4 & 0 & 0 \\
\hline \multicolumn{9}{|l|}{$15 \times 10$} \\
\hline la21 & 2181 & 2066 & 1856 & 1764 & 3639 & 3123 & 2787 & 2688 \\
\hline la22 & 2616 & 2360 & 2045 & 1862 & 4061 & 3798 & 3511 & 3318 \\
\hline la23 & 2701 & 2457 & 2200 & 2120 & 4942 & 4128 & 3642 & 3537 \\
\hline la24 & 2344 & 2227 & 1975 & 1882 & 4265 & 3915 & 3398 & 3268 \\
\hline la25 & 2129 & 2038 & 1766 & 1655 & 3742 & 3162 & 2738 & 2598 \\
\hline \multicolumn{9}{|l|}{$20 \times 10$} \\
\hline la26 & 8053 & 7117 & 6377 & 6163 & 13191 & 11945 & 11180 & 10260 \\
\hline la27 & 8533 & 7447 & 6584 & 6347 & 13281 & 12790 & 11453 & 10769 \\
\hline la28 & 7418 & 6718 & 6340 & 6217 & 12965 & 12169 & 10973 & 9621 \\
\hline la29 & 6898 & 5821 & 5496 & 5246 & 12067 & 11367 & 9746 & 9315 \\
\hline la30 & 6453 & 5856 & 5337 & 5224 & 10617 & 9783 & 8770 & 7379 \\
\hline \multicolumn{9}{|l|}{$30 \times 10$} \\
\hline la31 & 28484 & 25177 & 23913 & 21857 & 41804 & 41747 & 41090 & 39088 \\
\hline la32 & 38955 & 32441 & 27292 & 25136 & 45338 & 44723 & 44251 & 42510 \\
\hline la33 & 37945 & 35852 & 32246 & 28101 & 40062 & 39894 & 39164 & 36088 \\
\hline la34 & 43523 & 41059 & 36426 & 33874 & 42488 & 41882 & 41381 & 40125 \\
\hline la35 & 27341 & 25163 & 24507 & 23735 & 42060 & 41296 & 40418 & 38181 \\
\hline \multicolumn{9}{|l|}{$15 \times 15$} \\
\hline la36 & 1556 & 986 & 717 & 602 & 2302 & 1754 & 1338 & 1078 \\
\hline la37 & 1744 & 1184 & 932 & 822 & 2555 & 1978 & 1664 & 1377 \\
\hline la38 & 1451 & 1069 & 903 & 726 & 1984 & 1639 & 1099 & 726 \\
\hline la39 & 1467 & 813 & 561 & 405 & 1383 & 1075 & 786 & 630 \\
\hline la40 & 3458 & 1211 & 983 & 943 & 2195 & 1768 & 1204 & 1113 \\
\hline
\end{tabular}




\section{References}

Applegate D, Cook W (1991) A computational study of the job-shop scheduling problem. ORSA Journal on Computing 3(2):149-156

Balas E, Simonetti N, Vazacopoulos A (2008) Job shop scheduling with setup times, deadlines and precedence constraints. Journal of Scheduling 11(4):253-262

Blazewicz J, Domschke W, Pesch E (1996) The job shop scheduling problem: conventional and new solution techniques. European Journal of Operational Research 93(1):1-33

Brucker P, Knust S (2011) Complex Scheduling, 2nd edn. Springer

Brucker P, Thiele O (1996) A branch \& bound method for the general-shop problem with sequence dependent setup-times. OR Spectrum 18(3):145-161

Brucker P, Jurisch B, Sievers B (1994) A branch and bound algorithm for the job-shop scheduling problem. Discrete Applied Mathematics 49(1):107-127

Bülbül K, Kaminsky P (2013) A linear programming-based method for job shop scheduling. Journal of Scheduling 16(2):161-183

Bürgy R (2014) Complex Job Shop Scheduling: A General Model and Method. PhD thesis, Department of Informatics, University of Fribourg

Bürgy R, Gröflin H (2016) The blocking job shop with rail-bound transportation. Journal of Combinatorial Optimization 31(1):151-181

Eilon S, Hodgson R (1967) Job shops scheduling with due dates. International Journal of Production Research 6(1):1-13

Glover FW, Laguna M (1997) Tabu search. Kluwer

Gonçalves JF, Resende MGC (2014) An extended Akers graphical method with a biased random-key genetic algorithm for job-shop scheduling. International Transactions in Operational Research 21(2):215-246

González MA, Vela CR, Sierra M, Varela R (2010) Tabu search and genetic algorithm for scheduling with total flow time minimization. In: COPLAS 2010: ICAPS Workshop on Constraint Satisfaction Techniques for Planning and Scheduling Problems, pp 33-41

González MÁ, González-Rodríguez I, Vela CR, Varela R (2012a) An efficient hybrid evolutionary algorithm for scheduling with setup times and weighted tardiness minimization. Soft Computing 16(12):2097-2113

González MA, Vela CR, Varela R (2012b) A competent memetic algorithm for complex scheduling. Natural Computing 11(1):151-160

Graham RL, Lawler EL, Lenstra JK, Rinnooy Kan AHG (1979) Optimization and approximation in deterministic sequencing and scheduling: a survey. Annals of Discrete Mathematics 5:287-326

Grimes D, Hebrard E (2015) Solving variants of the job shop scheduling problem through conflict-directed search. INFORMS Journal on Computing 27(2):268-284

Gröflin H, Klinkert A (2007) Feasible insertions in job shop scheduling, short cycles and stable sets. European Journal of Operational Research 177(2):763-785

Gröflin H, Klinkert A (2009) A new neighborhood and tabu search for the blocking job shop. Discrete Applied Mathematics 157(17):3643-3655

Gröflin H, Pham DN, Bürgy R (2011) The flexible blocking job shop with transfer and set-up times. Journal of Combinatorial Optimization 22(2):121-144 
Hooker JN (1995) Testing heuristics: We have it all wrong. Journal of Heuristics 1(1):33-42

Lawrence S (1984) Supplement to resource constrained project scheduling: an experimental investigation of heuristic scheduling techniques. GSIA, Carnegie Mellon University, Pittsburgh, PA

Mascis A, Pacciarelli D (2002) Job-shop scheduling with blocking and no-wait constraints. European Journal of Operational Research 143(3):498-517

Mati Y, Dauzère-Pérès S, Lahlou C (2011) A general approach for optimizing regular criteria in the job-shop scheduling problem. European Journal of Operational Research 212(1):33-42

Nowicki E, Smutnicki C (1996) A fast taboo search algorithm for the job shop problem. Management Science 42(6):797-813

Nowicki E, Smutnicki C (2005) An advanced tabu search algorithm for the job shop problem. Journal of Scheduling 8(2):145-159

Oddi A, Rasconi R, Cesta A, Smith SF (2012) Iterative improvement algorithms for the blocking job shop. In: Twenty-Second International Conference on Automated Planning and Scheduling

Peng B, Lü Z, Cheng TCE (2015) A tabu search/path relinking algorithm to solve the job shop scheduling problem. Computers and Operations Research 53:154-164

Perregaard M, Clausen J (1998) Parallel branch-and-bound methods for the job-shop scheduling problem. Annals of Operations Research 83(0):137-160

Pinedo ML (2012) Scheduling: Theory, Algorithms, and Systems, 4th edn. Springer

Potts CN, Strusevich VA (2009) Fifty years of scheduling: a survey of milestones. Journal of the Operational Research Society 60:S41-S68

Pranzo M, Pacciarelli D (2015) An iterated greedy metaheuristic for the blocking job shop scheduling problem. Journal of Heuristics

Taillard E (1994) Parallel taboo search techniques for the job shop scheduling problem. ORSA Journal on Computing 6:108-108

Tufte ER (2001) The Visual Display of Quantitative Information, 2nd edn. Bertrams

Vela C, Varela R, González M (2010) Local search and genetic algorithm for the job shop scheduling problem with sequence dependent setup times. Journal of Heuristics 16:139-165

Zhang CY, Li P, Rao Y, Guan Z (2008) A very fast TS/SA algorithm for the job shop scheduling problem. Computers and Operations Research 35(1):282-294 